Bayesian inverse problems for functions and applications to fluid mechanics

This article has been downloaded from IOPscience. Please scroll down to see the full text article.

2009 Inverse Problems 25115008

(http://iopscience.iop.org/0266-5611/25/11/115008)

View the table of contents for this issue, or go to the journal homepage for more

Download details:

IP Address: 137.205.57.217

The article was downloaded on 16/02/2011 at 11:38

Please note that terms and conditions apply. 
[80]

S.L. Cotter, M. Dashti, J.C. Robinson and A.M. Stuart, Bayesian inverse problems for functions and applications to fluid mechanics.

Inverse Problems 25 (2009)

115008. doi: $10.1088 / 0266-5611 / 25 / 11 / 115008$. 


\title{
Bayesian inverse problems for functions and applications to fluid mechanics
}

\author{
S L Cotter, M Dashti, J C Robinson and A M Stuart \\ Mathematics Institute, University of Warwick, Coventry CV4 7AL, UK
}

Received 13 June 2009, in final form 10 September 2009

Published 29 October 2009

Online at stacks.iop.org/IP/25/115008

\begin{abstract}
In this paper we establish a mathematical framework for a range of inverse problems for functions, given a finite set of noisy observations. The problems are hence underdetermined and are often ill-posed. We study these problems from the viewpoint of Bayesian statistics, with the resulting posterior probability measure being defined on a space of functions. We develop an abstract framework for such problems which facilitates application of an infinite-dimensional version of Bayes theorem, leads to a well-posedness result for the posterior measure (continuity in a suitable probability metric with respect to changes in data), and also leads to a theory for the existence of maximizing the posterior probability (MAP) estimators for such Bayesian inverse problems on function space. A central idea underlying these results is that continuity properties and bounds on the forward model guide the choice of the prior measure for the inverse problem, leading to the desired results on well-posedness and MAP estimators; the PDE analysis and probability theory required are thus clearly dileneated, allowing a straightforward derivation of results. We show that the abstract theory applies to some concrete applications of interest by studying problems arising from data assimilation in fluid mechanics. The objective is to make inference about the underlying velocity field, on the basis of either Eulerian or Lagrangian observations. We study problems without model error, in which case the inference is on the initial condition, and problems with model error in which case the inference is on the initial condition and on the driving noise process or, equivalently, on the entire time-dependent velocity field. In order to undertake a relatively uncluttered mathematical analysis we consider the two-dimensional Navier-Stokes equation on a torus. The case of Eulerian observationsdirect observations of the velocity field itself-is then a model for weather forecasting. The case of Lagrangian observations-observations of passive tracers advected by the flow-is then a model for data arising in oceanography. The methodology which we describe herein may be applied to many other inverse problems in which it is of interest to find, given observations, an infinitedimensional object, such as the initial condition for a PDE. A similar approach might be adopted, for example, to determine an appropriate mathematical
\end{abstract}


setting for the inverse problem of determining an unknown tensor arising in a constitutive law for a PDE, given observations of the solution. The paper is structured so that the abstract theory can be read independently of the particular problems in fluid mechanics which are subsequently studied by application of the theory.

\section{Introduction}

The explosion of data gathering over the last few decades has been, and will continue to be, phenomenal [35]. There has also been a massive change in the scale of computational power which is now routinely available. These technology-driven changes are leading to both the need for inference on massive and highly heterogeneous data sets, and for the possibility of modelling ever more complex structures and systems. Making sense of data, in the context of the modelling of complex systems, is a very challenging task. The field of statistics provides a rational basis for the analysis of data. On the other hand, in many application areas, there is an enormous amount of information in the form of sophisticated mathematical models, often developed over decades or centuries. As a consequence of these advances in technology, applied mathematics and statistics are required to work in concert in order to significantly progress understanding. Blending these two view points-statistics, which is data driven, and mathematical modelling which provides systematic predictive tools, often in the form of partial differential equations - leads to challenging problems related to the precise specification of a mathematical problem, its analysis and the development of computational tools for its study.

In many applications the unknown, which we wish to determine from the mathematical model and the data, is a function, whilst the data set is finite and noisy. The problem is hence underdetermined and is often ill-posed. It may be viewed as an inverse problem. Bayesian statistics provides a useful viewpoint for the study of such problems and we choose to adopt this approach [39] $]^{1}$. The specific motivation for our paper is to develop the Bayesian viewpoint on function space and to demonstrate, by means of applications arising in fluid mechanics, that the abstract machinery developed can be applied to concrete applications.

When developing the abstract framework we will consider $x$ to be the function which we wish to determine and $y$ the data which we have available to us. We assume that, in the idealized setting in the absence of observational noise, the function $x$ is mapped to the data $y$ by an observation operator $\mathcal{G}: y=\mathcal{G}(x)$; this operator is formed from composition of the forward model with its projection onto the observed data ${ }^{2}$. Using this operator, and an understanding of the statistics of the observational noise $\sigma$ which inevitably pollutes the observations in many applications so that $y=\mathcal{G}(x)+\sigma$, it is typically straightforward to write the likelihood of the data: the probability that we obtain the data, given the function $x$. We denote this by $\mathbb{P}(y \mid x)^{3}$. Note, however, that we do not know $x$ : it is the function we wish to determine. Instead we assume that we may encode our knowledge about $x$, in the absence of data, in a prior probability distribution $\mathbb{P}(x)$. If $x$ was finite-dimensional, Bayes theorem would give the posterior probability of the function $x$ given the data $y$, which we denote by $\mathbb{P}(x \mid y)$, via the formula ${ }^{4}$

1 We recognize, however, that there are serious philosophical issues associated with the viewpoint, as well as non-trivial practical issues associated with quantifying lack of knowledge about a system in terms of probability distributions.

2 We depart from the widely used notation in [37] since the notation therein conflicts with notation required for the PDE setting adopted here.

3 The notation $\mathbb{P}(\cdot)$ will be used consistently to denote a probability; $\mathbb{E}(\cdot)$ will be used to denote an expectation.

4 Here, and in what follows, $\propto$ denotes 'is proportional to'. 


$$
\frac{\mathbb{P}(x \mid y)}{\mathbb{P}(x)} \propto \mathbb{P}(y \mid x) .
$$

In the infinite-dimensional case there is no density with respect to the Lebesgue measure. In this case, Bayes rule should be interpreted as providing the Radon-Nikodym derivative between the posterior measure $\mu^{y}(\mathrm{~d} x)=\mathbb{P}(\mathrm{d} x \mid y)$ and the prior measure $\mu_{0}(\mathrm{~d} x)=\mathbb{P}(\mathrm{d} x)$ :

$$
\frac{\mathrm{d} \mu^{y}}{\mathrm{~d} \mu_{0}}(x) \propto \rho(y-\mathcal{G}(x)),
$$

where $\rho$ is the pdf for $\sigma$.

If the Bayesian framework is adopted ${ }^{5}$ then it is arguable that the posterior probability $\mathbb{P}(x \mid y)$ is the answer to the problem of combining observations and model. It is thus an important object. However, whilst this conceptual development is straightforward, it raises many interesting mathematical and computational challenges. The purpose of this paper is to address several mathematical problems related to the posterior probability distribution. In particular we will study the following three questions: (a) under what conditions on the observation operator and the prior probability distribution can we find a function-space valued version of the Bayes theorem?; (b) when is the posterior measure continuous with respect to the data, and in what metric for measures should this continuity be understood?; (c) when are maximum a posteriori (MAP) estimators, given by

$$
\operatorname{argmax}_{x} \mathbb{P}(x \mid y),
$$

well defined in the function-space context? Partial answers to these three questions may be found in theorem 2.1 (see also corollary 2.2) and theorems 2.5 and 2.7. Another important question is (d) how can we exploit the definition of the posterior on function space in the design of sampling methods to probe $\mathbb{P}(x \mid y)$ given by (1.2)? This question is addressed in the paper [13].

Although we will illustrate the abstract theory in the context of problems from fluid mechanics, the methodology which we introduce in this paper may be applied to many other inverse problems in which it is of interest to find, given observations, an infinitedimensional object, such as the initial condition for a PDE. A similar approach might be adopted, for example, to determine an appropriate mathematical setting for the inverse problem of determining an unknown tensor arising in a constitutive law for a PDE, given observations of the solution. Overview of a range of nonlinear inverse problems arising in PDE may be found in the paper [8] and for geological inverse problems in [24, 25]. The basic approach that we adopt in this paper may be viewed as the analogue for inverse problems of the wellposedness framework for forward problems. The essential ingredients are (i) the identification of an observation operator, mapping unknown functions to the data, in the absence of noise; (ii) derivation of bounds and Lipschitz properties (and sometimes differentiability) of the observation operator, in appropriate function spaces; (iii) specification of the prior measure to ensure sufficient regularity of the observation operator in order to define the posterior measure and obtain continuity of the measure with respect to changes in data; (iv) determination of the properties of the posterior that are useful in the definition of efficient computational methods for probing the posterior measure, including MAP estimators, and Markov-Chain Monte Carlo (MCMC) methods [61].

\subsection{Literature in Bayesian inverse problems}

The book [39] provides an excellent introduction to the Bayesian approach to inverse problems, especially large inverse problems arising in differential equations. The approach taken there is

5 There are nontrivial questions here associated with objective determination of the priors [64]. 
to first discretize the problem and then apply the Bayesian methodology to a finite-dimensional problem; the idea of trying to capture the limit of infinite resolution is addressed by use of statistical extrapolation techniques based on modelling the error from finite-dimensional approximation [41]. In contrast, the book [66] directly advocates the viewpoint taken in this paper, namely that it can be valuable to formulate inverse problems on function space (see chapter 5 in particular); however the problems studied there are primarily Gaussian, whereas the theory we develop is not limited in this way. The book [59] is devoted entirely to statistical problems for functions, although the treatment is not Bayesian, and the models are somewhat simpler than those arising from PDE problems in the applications which motivate our work; also the function space setting does not play a major role in the approach taken there. There is some literature concerning the Bayesian viewpoint for linear inverse problems on function space, including the early study [27]. A more recent paper, with references to some of the current literature, is [36]. The papers [48, 49] also study Bayesian inversion for linear inverse problems on function space; they introduce the notion of discretization invariance and investigate the question of whether it is possible to derive regularizations of families of finite-dimensional problems, in a fashion which ensures that meaningful limits are obtained. In contrast, our viewpoint is to define the inverse problem directly on function space; discretization invariance is then guaranteed for finite-dimensional approximations of the function space inverse problem. Furthermore, our approach is not limited to linear observation operators.

The regularized least squares approach to inverse problems, which underlies the MAP estimator (also known as the variational approach) is widely studied in the infinite-dimensional context. For the Hilbert spaces setting see the book [21] and the references therein; for the Banach space setting see the recent papers [34, 42, 52] and the references therein. Although we concentrate in this paper on Gaussian priors, and hence on regularization via addition of a quadratic penalization term, there is active research in the use of different regularizations [34, 42, 49, 52]. In particular, the use of total variation based regularization, and related wavelet based regularizations, is central in image processing [63], and is hence an object of some interest.

The use of MCMC methods for sampling complex high-dimensional posterior distributions is, although extremely computationally challenging, starting to become feasible; recent examples of work in this direction include [11, 20, 40]. In a companion paper [13] we show that the abstract framework developed in this paper, and exploited here to prove well posedness of the posterior measure and well definedness of the MAP estimator in infinite dimensions, is also useful for the definition, analysis and implementation of efficient MCMC methods for sampling the posterior measure on an infinite-dimensional space. The resulting MCMC methods that arise are non-standard generalizations of methods such as the random walk metropolis and metropolis-adjusted Langevin algorithms which are widely used in the finite-dimensional setting [61]; it is shown in $[8,9]$ that these non-standard extensions lead to algorithms which scale well under mesh refinement, in contrast to the standard algorithms as analysed in $[8,61]$.

\subsection{Literature in fluid mechanics}

In fluid dynamical applications the blending of data and models, particularly in the timedependent context, is referred to as data assimilation. The subject has been systematically developed over the last few decades and has had measurable impact on the efficacy of, for example, weather forecasting. This may be considered to be one of the major achievements in applied computational PDE from the last half century. In the context of numerical weather 
prediction the text [44] overviews much of the recent literature, whilst for oceanographic applications the text [5] plays a similar role. See also the papers [38, 47, 50, 54] for some representative examples related to the specific applied problems that we will consider later in this paper. Recent mathematical overviews of the subject of data assimilation in fluid mechanics include $[3,53]$.

There are two major difficulties facing the research community in data assimilation for fluid mechanics applications: the first is that, at currently available levels of computational capability, it is often impossible to resolve all the desired physics and hence sub-grid scale modelling plays an important role; the second is that the resulting dynamical models are very high dimensional, as they are approximations to PDEs. The need to deal with the first issue leads to increased demands for resolution, as computer power grows, simultaneously increasing the problems arising from the second issue. The situation is further complicated by the presence of a plethora of data, or varying quality, which must be optimally blended with the computational models, placing further demands on computational resources. The data can, in principle, be used to help quantify uncertainty in predictions from PDE models. This leads to a major question confronting the field: should any increase in available computer resources be used to increase resolution of the computational model, or should it be used to improve estimates of uncertainty? This important question is discussed, in the context of climate modelling, in [57].

The work in this paper does not provide answers to this difficult question. However having the notion of an ideal solution to the problem of blending data and model in the limit of infinite resolution, which we assume to be the posterior measure given by (1.2), is a crucial conceptual building block. In short term, this ideal solution can be used as a benchmark against which to evaluate, and improve, practical computational methodologies which are used on large-scale applications because of constraints arising from finite resources. In the longer term, algorithms may even move towards attempts to directly compute the ideal solution. In order to explain this perspective we now describe how the ideal solution (1.2) relates to current computational practice in the field of data assimilation for fluid mechanics.

There are two competing (although sometimes overlapping) methodologies that dominate computational practice: filtering methods and variational methods. A good overview of these topics, aimed at applications in geophysical fluid dynamics, may be found in the book [23]. A second important methodological distinction to make is between forecasting methods, which are typically used online to make predictions, and hindcasting methods which are used offline to obtain improved understanding (sometimes termed reanalysis) and, for example, may be used for the purposes of parameter estimation to obtain improved models. It is important to realize that the subgrid scale models referred to earlier often have parameters in them which are not directly physically measurable and so parameter estimation can play an important role in their identification; reanalysis is hence potentially quite important.

Filtering methods solve a different problem from that giving rise to (1.1). They are based on the assumption that the data are acquired sequentially in time and ordered as $y=\left\{y_{i}=y\left(t_{i}\right)\right\}_{i=1}^{m}$ with $i$ indexing the time $t_{i}$ at which data are acquired. If $x=\left\{x_{i}=x\left(t_{i}\right)\right\}_{i=1}^{m}$ denotes the solution of the desired dynamical model then filtering is aimed at finding the sequence of probability measures

$$
\mathbb{P}_{i}=\mathbb{P}\left(x_{i} \mid\left\{y_{j}\right\}_{j=1}^{i}\right) .
$$

Clearly $\mathbb{P}_{m}$ agrees with the marginal distribution of $\mathbb{P}(x \mid y)$ from (1.1) at time $t=t_{m}$; however the marginal distribution at other times $t_{i}<t_{m}$ will differ from $\mathbb{P}_{i}$. Filtering is important for two reasons: first it may be used in online situations; and second it breaks down the 
computational cost associated with the dimension of time, which is treated in a single block in (1.1), but is broken down sequentially in the filtering approach.

In practice filtering in high-dimensional systems is extremely hard, and various forms of approximation are employed to deal with this issue. A widely used approach is that based on the ensemble Kalman filter [23] which uses an ensemble of particles to propagate the dynamics, but incorporates data using a Gaussian approximation which is hard to justify in general (see also [7, 56]). The paper [4] describes a generalization of the ensemble Kalman filter, based on mixtures of Gaussians, motivated by the high-dimensional systems arising in fluid dynamics data assimilation problems. The paper [6] studies the use of filtering techniques in high dimensions, motivated by oceanographic data assimilation, and contains a study of the question of how to define families of finite-dimensional filters which converge to a function space valued limit as the finite-dimensional computation is refined; it is thus related to the concept of discretization invariance referred to earlier and now starting to undergo systematic mathematical study. However, the methodology for proving limiting behaviour in [6], based on Fourier analysis, is useful only for linear Gaussian problems. Furthermore results similar to those in [6] could be obtained by attempting to directly define a Gaussian measure on function space and using ideas similar to those underlying the proof of lemma 4.3 to establish the desired regularity; in this paper we use the same lemma to understand the regularity of functions drawn from our prior Gaussian measures.

In theorems 3.4, 3.8, 3.11 and 3.14 we show that the ideal solution (1.2) is well defined for a variety of problems arising in fluid mechanics which lead to non-Gaussian posteriors; furthermore we show that these idealized problems satisfy assumptions 2.4 meaning that, by theorem 2.5 , the idealized solution responds continuously to changes in the data. By marginalization, as described above, these ideal smoothing solutions also apply to the solution of the filtering problem on function space, at the final observation time, and hence establish existence and continuity with respect to data of the filter at the final time, under appropriate prior assumptions. Similar ideas could also be used to study the existence and continuity of the filter distribution at times prior to the last observation time.

What, then, is the potential role to be played by the ideal solution (1.2) in the understanding of filtering? In addition to defining clearly the ideal solution, in the general nonlinear nonGaussian case and in the limit of infinite mesh resolution, in the short term this ideal solution can be used to compute (a good quality approximation to) the ideal answer, which can then be marginalized to the final time coordinate, in order to test and guide the development of filtering methods. In [13] we show that it is within computational reach to compute such ideal solutions for simple model problems arising in the PDEs of fluid mechanics; in particular we study the two-dimensional Stokes equations with Eulerian or Lagrangian data (leading in the latter case to posterior measures which are non-Gaussian) by use of MCMC methods. In $[1,2]$ we include preliminary studies comparing the ideal solution with ensemble Kalman filter methods applied to Lagrangian data driven by a low-dimensional truncation of the linearized shallow water equations. An unambiguous and mathematically well-defined definition of the ideal solution plays an important role in underpinning such computational studies.

Variational methods take a different approach from that employed in filtering. In essence they are based around finding the MAP estimator (1.3) [65]. In the data assimilation community this is done both for data distributed in space time [19] and for data in a short time window [15]. The recent paper [51] investigates relationships between the variational method, unpredictability of the dynamical model and statistical effects. In the absence of what we term model error, the variational method we study in this paper is known as ADVAR; in the presence of model error it is known as weak constraint 4DVAR. Calculating the MAP estimator for the time-dependent PDEs of fluid mechanics, in the presence of finite data distributed in 
time leads to boundary value problems on a space-time domain, driven by source terms at the data points. The book [5] provides a very clear discussion of variational methods and, of particular relevance to the development in this paper, develops a view of the subject in function space. This development is most complete in the case where (1.2) is Gaussian so that the PDEs defining the optimal solution can be solved analytically. The book discusses iterative methods which can be used in the non-Gaussian case arising when the observation operator $\mathcal{G}$ is nonlinear (see section 3.3 of [5]), but does not provide an answer to the question of existence of a probability maximizer, in the appropriate function space defined by the prior covariance operator, in the non-Gaussian case. The existence of such maximizers, using techniques from the calculus of variations, is undertaken in [68] for Burgers equations and in [55] for a model of ocean circulation including velocity, temperature and salinity variables. An alternative approach is to study the existence of solutions to the resulting Euler-Lagrange equations, and this is undertaken for a data assimilation model based on the vorticity equation in [30]; note however that the proof of existence of a solution in that paper requires a sufficiently short time interval. In contrast the method we employ to prove the existence of minimizers, resulting in theorem 2.7, does not require this. Indeed in theorems 3.4, 3.8, 3.11 and 3.14, we show that the ideal solution (1.2) is well defined for a variety of problems arising in fluid mechanics with data on arbitrary finite time intervals and that, in each case, the ideal solution satisfies assumptions 2.4 meaning that theorem 2.7 applies so that a MAP estimator exists. These techniques could be extended to the PDE model arising in [30] under an appropriate leastsquares formulation dictated by a Gaussian prior measure on the initial condition (and possibly forcing). Note, however, that theorem 2.7 does not address the question of multiple minimizers: it simply asserts that a global minimizer exists. In practical applications, especially if the time interval is long and/or the growth of linear perturbations to the dynamical model is strong, many other local minimizers may exist.

What does study of the ideal solution (1.2) add to our understanding of variational methods? The first thing that this viewpoint adds is an answer to the question of the existence of probability maximizers, in the general non-Gaussian case, on function space. This enables us to conclude that proper specification of the prior measures leads to a properly specified problem for the probability maximizer, on function space. This gives a framework which satisfies the desirable property that successive mesh refinements will lead to probability maximizers which converge, since we establish conditions under which the function space limit is well defined. Here we generalize the approach employed in [55] to prove the existence of probability maximizers in function space. A second thing added to our understanding of variational methods is that the ideal solution (1.2) provides the right object against which to compare variational methods. The latter are useful only when the posterior measure $\mathbb{P}(x \mid y)$ is strongly peaked at a single point (a Dirac mass) or is unimodal and well approximated by a Gaussian; in the Gaussian case additional covariance information will need to be extracted as well as the maximizer. As for filtering methods, we now have the opportunity to compute (a good quality approximation to) the ideal answer in order to understand situations in which variational approximations are accurate and in which they are not. For example, if the posterior is multimodal, the variational method can be quite misleading. In [13] we show that it is indeed possible to compute probability distribution arising from the ideal solution (1.2) on some simple model PDE problems arising from the Stokes equations, with both Eulerian data (leading to a Gaussian posterior) and Lagrangian data (leading to a non-Gaussian posterior).

In summary, then, the approach we adopt in this paper provides a mathematical framework for the evaluation of methods widely used in practice, such as filtering and variational methods, both of which should be viewed as giving approximations to the ideal solutions which is the posterior probability measure. This will enable comparison of such methods with the ideal 
answer to the problem of cojoining data and model. It is also conceivable that, as computer power grows, it will be worth investing in direct MCMC-based methods to sample the ideal posterior distribution, and that this will give rise to new approaches to the problem of data assimilation in fluid mechanics. This may be useful for both analysis and forecasting and, perhaps more directly for reanalysis, parameter estimation in sub-grid scale models and systematic study of model error. In this context we define model error to be an error term in the dynamical model equations, as in [30], because this is both physically and mathematically natural; note however that in practical data assimilation, model (or representativity) errors are sometimes combined with the observations errors [14].

\subsection{Outline of the paper}

In section 2 we develop an abstract framework for Bayesian inversion on function space, given noisy data. In subsection 2.1 we introduce a version of the Bayes theorem in infinite dimensions, enabling us to show that the posterior measure is well defined and is absolutely continuous with respect to the prior ${ }^{6}$. In subsection 2.2 we study continuity of the posterior measure with respect to data, showing that small changes in the data lead to small changes to the (Bayesian) solution of the inverse problem. We use the Hellinger metric to study changes in the measure as this allows proof of continuity of expectations of polynomially bounded functions. We thus establish a form of well posedness for the inverse problem. The same properties used to establish well posedness of the posterior measure are also useful for the definition, analysis and implementation of maximum a posteriori (MAP) estimators (also known as variational methods) on function space, a subject that we pursue in subsection 2.3. The entire section 2 is self-contained and independent of any specific applications to problems arising in fluid mechanics; it should be accessible to readers with general interest in Bayesian inversion on function space. Furthermore, whilst the proofs in section 2 may not be of direct interest to readers interested mostly in applications to fluid mechanics, or other inverse problems on function space, the basic statements of theorems 2.1, 2.5 and 2.7, as well as corollary 2.2 summarize the useful outcomes of the theoretical framework and may be read independently of the proofs.

In section 3 we demonstrate that the abstract theory may be applied to four concrete problems arising in fluid mechanics. The four subsections concern problems in which the data are Eulerian or Lagrangian and in which model error may or may not be present. Appendix 1, section 4, contains some basic results on the existence and uniqueness of solutions to the Navier-Stokes equations, and to the Lagrangian trajectories that it generates. The section also contains some background material required for the specification of Gaussian random field priors on functions of space alone (the initial condition) or space time (the forcing). The results from this section are summarized in a readable way at the start of section 3 , so that the reader interested primarily in applications to fluid mechanics can absorb the results of section 3 without reading section 4 . The results of section 3 also rely heavily on estimates satisfied by solutions of the Navier-Stokes equation, which are presented in a self-contained way in appendix 2, section 5, as they may be of independent interest; these results, however, are not required for the reader interested primarily in applications to fluid mechanics as they are required only for the proofs, not the statements, of results in section 3.

6 Absolute continuity is a measure theoretic concept ensuring that the posterior measure does not assign non-trivial probability to events which have probability zero under the prior; it should not be confused with standard continuity of measures with respect to small changes which we discuss in subsection 2.2. 


\section{Bayesian inversion on function space}

We describe an abstract Bayesian framework for inverse problems in which the unknown is a function and the data are finite. These problems are hence underdetermined. They are also frequently ill-posed in a classical sense. We describe three key ideas: (a) we prove a version of the Bayes theorem relevant to this function space setting, showing that the posterior measure is absolutely continuous with respect to the prior measure, and identifying the Radon-Nikodym derivative as the data likelihood; (b) we demonstrate a form of well posedness by proving that the posterior measure is Lipschitz continuous in the data, when the Hellinger metric is used as a metric on the posterior measure; (c) we show that the MAP estimator for the posterior measure (the posterior probability maximizer) is well defined whenever the posterior measure is, by using techniques from the calculus of variations (or optimal control).

Throughout the following we denote by $\langle\cdot, \cdot\rangle$ the standard Euclidean scalar product on $\mathbb{R}^{m}$, which induces the standard Euclidean norm $|\cdot|$. We also define ${ }^{7}\langle\cdot, \cdot\rangle_{C}:=\left\langle C^{-\frac{1}{2}} \cdot, C^{-\frac{1}{2}} \cdot\right\rangle$ for any positive definite symmetric matrix $C$; this induces the norm $|\cdot|_{C}:=\left|C^{-\frac{1}{2}} \cdot\right|$. We will also use the notation $\langle\cdot, \cdot\rangle_{\mathcal{C}}$ and $|\cdot|_{\mathcal{C}}:=\left|\mathcal{C}^{-\frac{1}{2}} \cdot\right|$ to denote a new norm and inner product, given a Hilbert space $(X,\langle\cdot, \cdot\rangle)$ and a trace class, positive and self-adjoint operator $\mathcal{C}$. When $\mathcal{C}$ is a covariance operator of a Gaussian measure this new Hilbert space is termed the CameronMartin space [10], denoted by $E$. It is important because it is precisely the space in which shifts of the mean of the Gaussian law give rise to mutually absolutely continuous measures; however it has measure zero under the Gaussian measure itself [10, 17]. The space also arises naturally when studying MAP estimators (variational methods).

\subsection{Bayes theorem for functions}

Let $x$ denote an unknown function that we wish to determine from a finite-dimensional vector $y$ of observations. We assume that the observations are related to the unknown function $x$ by

$$
y=\mathcal{G}(x)+\sigma .
$$

Here $\sigma$ is an unbiased observational noise which, for simplicity, we take to follow the Gaussian law $\mathcal{N}(0, \Sigma)^{8}$. The function $\mathcal{G}$ is the observation operator which maps the unknown functions into the data when no observational noise $\sigma$ is present.

We develop a version of the Bayes theorem on function space, in order to find an expression for the probability distribution of the desired function $x$, given the observations $y$. We assume that $x$ is in a Banach space $\left(X,\|\cdot\|_{X}\right)$ and that $y \in \mathbb{R}^{m}$. We define $\Phi: X \times \mathbb{R}^{m} \rightarrow \mathbb{R}$ by

$$
\Phi(x ; y)=\frac{1}{2}|y-\mathcal{G}(x)|_{\Sigma}^{2} .
$$

The likelihood of the data is

$$
\mathbb{P}(y \mid x) \propto \exp (-\Phi(x ; y))
$$

since $\sigma \sim \mathcal{N}(0, \Sigma)^{9}$. In the case where $x$ is finite dimensional, Bayes' theorem [58] leads to the conclusion that the ratio of the posterior distribution $\mathbb{P}(x \mid y)$ to the prior distribution $\mathbb{P}(x)$ is

$$
\frac{\mathbb{P}(x \mid y)}{\mathbb{P}(x)} \propto \mathbb{P}(y \mid x) \propto \exp (-\Phi(x ; y)) .
$$

In the case where $x$ is infinite dimensional there is no density with respect to Lebesgue measure. However, the ratio of the posterior to prior measures can be given a meaning and

\footnotetext{
7 We use the symbol $:=$ to denote a quantity defined by the identity.

8 We use $\mathcal{N}(m, C)$ to denote a Gaussian measure with mean $m$ and covariance matrix/operator $C$.

9 Here, and elsewhere in the paper, $\sim$ is used for 'is distributed according to'.
} 
the natural analogue of the finite-dimensional result is as follows: we expect that the posterior $\mu^{y}(\mathrm{~d} x)=\mathbb{P}(\mathrm{d} x \mid y)$ is absolutely continuous with respect to the prior $\mu_{0}(\mathrm{~d} x)=\mathbb{P}(\mathrm{d} x)$ and that the Radon-Nikodym derivative is given by

$$
\frac{\mathrm{d} \mu^{y}}{\mathrm{~d} \mu_{0}}(x) \propto \exp (-\Phi(x ; y)) \text {. }
$$

The following theorem and corollary give simple conditions under which this is indeed the case. They may be viewed as generalizations of the Bayes theorem to the infinite-dimensional setting.

Theorem 2.1. If $\mathcal{G}: X \rightarrow \mathbb{R}^{m}$ is $\mu_{0}$-measurable then the posterior measure $\mu^{y}(\mathrm{~d} x)=\mathbb{P}(\mathrm{d} x \mid y)$ is absolutely continuous with respect to the prior measure $\mu_{0}(\mathrm{~d} x)$ and has the Radon-Nikodym derivative given by (2.2).

Proof. Let $\mathbb{Q}_{0}(\mathrm{~d} y)$ denote the $\mathbb{R}^{m}$-valued Gaussian measure $\mathcal{N}(0, \Sigma)$ and $\mathbb{Q}(\mathrm{d} y \mid x)$ the $\mathbb{R}^{m}$-valued Gaussian measure $\mathcal{N}(\mathcal{G}(x), \Sigma)$. By construction

$$
\begin{aligned}
\frac{\mathrm{d} \mathbb{Q}_{1}}{\mathrm{~d} \mathbb{Q}_{0}}(y \mid x) & =\exp \left(-\frac{1}{2}|y-\mathcal{G}(x)|_{\Sigma}^{2}+\frac{1}{2}|y|_{\Sigma}^{2}\right) \\
& \propto \exp (-\Phi(x ; y))
\end{aligned}
$$

with constant of proportionality independent of $x$. Now define ${ }^{10}$

$$
\begin{aligned}
& v_{0}(\mathrm{~d} y, \mathrm{~d} x)=\mathbb{Q}_{0}(\mathrm{~d} y) \otimes \mu_{0}(\mathrm{~d} x), \\
& v(\mathrm{~d} y, \mathrm{~d} x)=\mathbb{Q}(\mathrm{d} y \mid x) \mu_{0}(\mathrm{~d} x) .
\end{aligned}
$$

The measure $\nu_{0}$ is clearly well defined by virtue of its product structure. Since $\mathcal{G}$ is $\mu_{0^{-}}$ measurable we deduce that $v$ is also well defined and is absolutely continuous with respect to $v_{0}$ with Radon-Nikodym derivative equal to that of $\mathbb{Q}$ with respect to $\mathbb{Q}_{0}$ :

$$
\frac{\mathrm{d} v}{\mathrm{~d} \nu_{0}}(\mathrm{~d} y, \mathrm{~d} x) \propto \exp (-\Phi(x ; y))
$$

the constant of proportionality is again independent of $x$. By lemma 2.3 (below) we have the desired result since, as $v_{0}$ is a product measure, $v_{0}(\mathrm{~d} x \mid y)=\mu_{0}(\mathrm{~d} x)$.

In many applications the observation operator is continuous on an appropriate Banach space $X$. The following corollary is hence a useful way of establishing the Bayes theorem in the form (2.2).

Corollary 2.2. If $\mathcal{G}: X \rightarrow \mathbb{R}^{m}$ is continuous and $\mu_{0}(X)=1$ then the posterior measure $\mu^{y}(\mathrm{~d} x)=\mathbb{P}(\mathrm{d} x \mid y)$ is absolutely continuous with respect to the prior measure $\mu_{0}(\mathrm{~d} x)$ and has Radon-Nikodym derivative given by (2.2).

Proof. Under the stated conditions we deduce that $\mathcal{G}$ is $\mu_{0}$ almost surely continuous, and is hence $\mu_{0}$ measurable $[22,43]$. Thus theorem 2.1 gives the desired result.

The following lemma, used in the proof of theorem 2.1, may be found in [22]. As stated here it is taken from [33], where it is used to prove a specific instance of theorem 2.1 arising in the study of conditioned diffusion processes, using the Gaussian Kalman-Bucy smoother measure from [32] as the reference measure.

Lemma 2.3. Consider two measurable spaces $(S, \mathcal{S})$ and $(T, \mathcal{T})$. Let $\mu$, v be probability measures on $S \times T$ and let $x: S \times T \rightarrow S$ and $y: S \times T \rightarrow T$ be the canonical projections.

${ }^{10}$ Here the symbol $\otimes$ is used to denote the (independent) product of two measures. 
Assume that $\mu$ has a density $\phi$ w.r.t. $v$ and that the conditional distribution $v_{x \mid y}$ exists. Then the conditional distribution $\mu_{x \mid y}$ exists and is given by

$$
\frac{\mathrm{d} \mu_{x \mid y}}{\mathrm{~d} v_{x \mid y}}(x)= \begin{cases}\frac{1}{c(y)} \phi(x, y), & \text { if } c(y)>0, \\ 1 & \text { else }\end{cases}
$$

with $c(y)=\int_{S} \phi(x, y) \mathrm{d} v_{x \mid y}(x)$ for all $y \in T$.

\subsection{Well-posed Bayesian inverse problems}

In the previous section we showed that Bayesian inverse problems for functions naturally give rise to a posterior probability measure $\mu^{y}(\mathrm{~d} x)$ given by (2.2). Here we work in a setting where the prior measure is Gaussian, and where $\mathcal{G}$ satisfies polynomial bounds in the space $X$. We show that even though the underlying classical inverse problem (inversion of $\mathcal{G}$ ) is underdetermined, and often ill-posed, the Bayesian inverse problem is well posed and that the posterior measure is Lipschitz in the data $y$. Furthermore we employ a metric on measures, the Hellinger metric, which implies continuity of expectations of all functions with second moments, with respect to the data.

We define the normalization constant

$$
Z(y)=\int_{X} \exp (-\Phi(x ; y)) \mathrm{d} \mu_{0}(x)
$$

so that the probability measure $\mu^{y}$ is given by

$$
\frac{\mathrm{d} \mu^{y}}{\mathrm{~d} \mu_{0}}(x)=\frac{1}{Z(y)} \exp (-\Phi(x ; y)) .
$$

If $\mathcal{G}: X \rightarrow \mathbb{R}^{m}$ is polynomially bounded and Lipschitz then it is straightforward to see that $\Phi$ given by (2.1) satisfies the following three properties:

Assumption 2.4. The function $\Phi: X \times \mathbb{R}^{m} \rightarrow \mathbb{R}$ satisfies the following:

- (i) there exists $p>0$ and for every $r>0 a K_{1}=K_{1}(r)>0$ such that, for all $x \in X$ and $y \in \mathbb{R}^{m}$ with $|y|<r$,

$$
0 \leqslant \Phi(x ; y) \leqslant K_{1}\left(1+\|x\|_{X}^{p}\right) ;
$$

- (ii) for every $r>0$ there is $K_{2}=K_{2}(r)>0$ such that, for all $u, v \in X$ and $y \in \mathbb{R}^{m}$ with $\max \left\{\left\|x_{1}\right\|_{X},\left\|x_{2}\right\|_{X},|y|\right\}<r$,

$$
\left|\Phi\left(x_{1} ; y\right)-\Phi\left(x_{2} ; y\right)\right| \leqslant K_{2}\left\|x_{1}-x_{2}\right\|_{X} ;
$$

- (iii) there is $q \geqslant 0$ and for every $r>0$ a $K_{3}=K_{3}(r)>0$ such that, for all $y_{1}, y_{2} \in \mathbb{R}^{m}$ with $\max \left\{\left|y_{1}\right|,\left|y_{2}\right|\right\}<r$, and for all $x \in X$,

$$
\left|\Phi\left(x ; y_{1}\right)-\Phi\left(x ; y_{2}\right)\right| \leqslant K_{3}\left(1+\|x\|_{X}^{q}\right)\left|y_{1}-y_{2}\right| .
$$

Note that it is continuity of $\Phi$, implied by the second condition on $\Phi$ in assumption 2.4 , which is implicitly used to prove the well definedness of the posterior measure in corollary 2.2. We now study continuity properties of the measure $\mu^{y}$ given by (2.5) with respect to the data $y$, under the first and third conditions of assumption 2.4. The total variation distance between $\mu$ and $\mu^{\prime}$ with common reference measure $v$ is

$$
d_{\mathrm{TV}}\left(\mu, \mu^{\prime}\right)=\frac{1}{2} \int\left|\frac{\mathrm{d} \mu}{\mathrm{d} \nu}-\frac{\mathrm{d} \mu^{\prime}}{\mathrm{d} \nu}\right| \mathrm{d} \nu .
$$


This is a commonly used metric ${ }^{11}$. We will employ a stronger metric ${ }^{12}$, given by the Hellinger distance between $\mu$ and $\mu^{\prime}$ and defined by

$$
d_{\mathrm{Hell}}\left(\mu, \mu^{\prime}\right)=\sqrt{\left(\frac{1}{2} \int\left(\sqrt{\frac{\mathrm{d} \mu}{\mathrm{d} \nu}}-\sqrt{\frac{\mathrm{d} \mu^{\prime}}{\mathrm{d} \nu}}\right)^{2} \mathrm{~d} \nu\right)} .
$$

Then [28]

$$
\frac{1}{\sqrt{2}} d_{\mathrm{TV}}\left(\mu, \mu^{\prime}\right) \leqslant d_{\mathrm{Hell}}\left(\mu, \mu^{\prime}\right) \leqslant d_{\mathrm{TV}}\left(\mu, \mu^{\prime}\right)^{\frac{1}{2}} .
$$

The Hellinger metric is natural because controlling it gives control over the calculation of expectations from a wide class. To be precise, assume that $f: X \rightarrow X$ has second moments with respect to both $\mu$ and $\mu^{\prime}$. Then the Hellinger metric has the following desirable property:

$$
\left\|\mathbb{E}^{\mu} f-\mathbb{E}^{\mu^{\prime}} f\right\| \leqslant 2\left(\mathbb{E}^{\mu}\|f\|^{2}+\mathbb{E}^{\mu^{\prime}}\|f\|^{2}\right)^{\frac{1}{2}} d_{\text {Hell }}\left(\mu, \mu^{\prime}\right) .
$$

Furthermore, if $f: X \rightarrow X$ has fourth moments then

$$
\left\|\mathbb{E}^{\mu} f \otimes f-\mathbb{E}^{\mu^{\prime}} f \otimes f\right\| \leqslant 2\left(\mathbb{E}^{\mu}\|f\|^{4}+\mathbb{E}^{\mu^{\prime}}\|f\|^{4}\right)^{\frac{1}{2}} d_{\text {Hell }}\left(\mu, \mu^{\prime}\right) .
$$

The following theorem proves Lipschitz continuity of the posterior measure with respect to changes in the data, in the Hellinger distance (and hence also in the total variation distance).

Theorem 2.5. $\quad$ Consider a measure $\mu^{y}$ which is absolutely continuous with respect to a measure $\mu_{0}$ with Radon-Nikodym derivative given by (2.5). Let assumptions 2.4 (i) and (iii) hold and assume that the prior measure $\mu_{0}$ is a Gaussian measure $\mathcal{N}(m, \mathcal{C})$ and that $\mu_{0}(X)=1$. Then the measure $\mu^{y}$ is Lipschitz in the data $y$, with respect to the Hellinger distance: if $\mu^{y}$ and $\mu^{y^{\prime}}$ are two measures given by (2.5) with data $y$ and $y^{\prime}$ then there is $C=C(r)>0$ such that, for all $y, y^{\prime}$ with $\max \left\{|y|,\left|y^{\prime}\right|\right\} \leqslant r$,

$$
d_{\mathrm{Hell}}\left(\mu^{y}, \mu^{y^{\prime}}\right) \leqslant C\left|y-y^{\prime}\right| .
$$

Consequently all polynomially bounded functions of $x \in X$ are continuous in $y$. In particular the mean and covariance operator are continuous in $y$.

Proof. Throughout the proof, all integrals are over $X$. The constant $C$ may depend upon $r$ and changes from occurrence to occurrence. Let $Z$ and $Z^{\prime}$ denote the normalization constants for $\mu^{y}$ and $\mu^{y^{\prime}}$ so that

$$
\begin{aligned}
Z & =\int \exp (-\Phi(x ; y)) \mathrm{d} \mu_{0}(x) \\
Z^{\prime} & =\int \exp \left(-\Phi\left(x ; y^{\prime}\right)\right) \mathrm{d} \mu_{0}(x) .
\end{aligned}
$$

Clearly

$$
|Z| \vee\left|Z^{\prime}\right| \leqslant 1
$$

since $\mu_{0}$ is a probability measure. Assume that $|y|,\left|y^{\prime}\right|<r$. Then, by assumptions 2.4 , we have

$$
|Z| \geqslant \int_{\|x\|_{X} \leqslant 1} \exp \left(-2 K_{1}(r)\right) \mathrm{d} \mu_{0}(x)=\exp \left(-2 K_{1}(r)\right) \mu_{0}\left(\|x\|_{X} \leqslant 1\right) .
$$

${ }^{11}$ It may be viewed as the $L^{1}$ norm on densities in the finite-dimensional setting where the reference measure is Lebesgue.

${ }^{12}$ Stronger in the sense implied by the inequalities (2.6). 
This is strictly positive because $\mu_{0}(X)=1$ and $\mu_{0}$ is Gaussian so that all balls in $X$ have positive measure [10]. We have an analogous lower bound for $\left|Z^{\prime}\right|$.

Since $\mu_{0}$ is Gaussian and $\|x\|_{X}$ is $\mu_{0}$-a.s. finite, it follows that all moments $\|x\|_{X}^{q}$ are $\mu_{0}$-a.s. finite by the Fernique theorem [10, 17]. Furthermore, using the fact that $\Phi(x ; \cdot)$ is Lipschitz, and since $\mu_{0}$ is a probability measure,

$$
\begin{aligned}
\left|Z-Z^{\prime}\right| & \leqslant \int K_{3}\left|y-y^{\prime}\right|\left(1+\|x\|_{X}^{q}\right) \mathrm{d} \mu_{0}(u) \\
& \leqslant C\left|y-y^{\prime}\right| .
\end{aligned}
$$

From the definition of Hellinger distance we have

$$
\begin{aligned}
2 d_{\mathrm{Hell}}\left(\mu, \mu^{\prime}\right)^{2} & =\int\left(Z^{-\frac{1}{2}} \exp \left(-\frac{1}{2} \Phi(x ; y)\right)-\left(Z^{\prime}\right)^{-\frac{1}{2}} \exp \left(-\frac{1}{2} \Phi\left(x ; y^{\prime}\right)\right)\right)^{2} \mathrm{~d} \mu_{0}(x) \\
& \leqslant I_{1}+I_{2}
\end{aligned}
$$

where

$$
\begin{aligned}
& I_{1}=\frac{2}{Z} \int\left(\exp \left(-\frac{1}{2} \Phi(x ; y)\right)-\exp \left(-\frac{1}{2} \Phi\left(x ; y^{\prime}\right)\right)\right)^{2} \mathrm{~d} \mu_{0}(x), \\
& I_{2}=2\left|Z^{-\frac{1}{2}}-\left(Z^{\prime}\right)^{-\frac{1}{2}}\right|^{2} \int \exp \left(-\Phi\left(x ; y^{\prime}\right)\right) \mathrm{d} \mu_{0}(x) .
\end{aligned}
$$
below,

Now, again using the Lipschitz property of $\Phi(x ; \cdot)$ and the fact that $\Phi$ is bounded from

$$
\begin{aligned}
\frac{Z}{2} I_{1} & \leqslant \int \frac{K_{3}}{4}\left|y-y^{\prime}\right|^{2}\left(1+\|x\|_{X}^{q}\right)^{2} \mathrm{~d} \mu_{0}(x) \\
& \leqslant C\left|y-y^{\prime}\right|^{2} .
\end{aligned}
$$

Also, using the bounds on $Z, Z^{\prime}$ from below,

$$
\begin{aligned}
\left|Z^{-\frac{1}{2}}-\left(Z^{\prime}\right)^{-\frac{1}{2}}\right|^{2} & \leqslant C\left(Z^{-3} \vee\left(Z^{\prime}\right)^{-3}\right)\left|Z-Z^{\prime}\right|^{2} \\
& \leqslant C\left|y-y^{\prime}\right|^{2}
\end{aligned}
$$

Combining gives the desired continuity result in the Hellinger metric.

Finally all moments of $\|x\|_{X}$ are finite under $\mu_{0}$ and hence under $\mu$ because the change of measure is bounded. The desired result follows from (2.7), (2.8).

Remark 2.6. It is natural to ask why we have asked for polynomial control in $x$, of the constants arising in assumption 2.4. In fact to obtain theorem 2.5 polynomial dependence can be replaced by dependence which grows as $\exp \left(\epsilon\|x\|_{X}^{2}\right)$, provided $\epsilon$ can be chosen arbitrarily small; this is all that is required to be able to apply the Fernique theorem [10,17] to obtain the required control: the Fernique theorem implies that, if $\mu_{0}(X)=1$, then for all $\epsilon$ sufficiently small,

$$
\int_{X} \exp \left(\epsilon\|x\|_{X}^{2}\right) \mu_{0}(\mathrm{~d} x)<\infty
$$

\subsection{Variational methods and optimal control}

In this section we address the question of finding $x$ to maximize the posterior probability given by (2.2), using methods from the calculus of variations. Finding $x$ to maximize the probability $\mu^{y}(d x)$ is referred to as a MAP estimator in the Bayesian statistics literature [39]. We provide an abstract theory for the existence of the MAP estimator, linked in a fundamental way to 
the natural assumptions 2.4 which imply that the posterior measure is well defined and well posed.

We work with probability measures $\mu^{y}$ given by $(2.2)$, with $\mu_{0}=\mathcal{N}(m, \mathcal{C})$ and $\Phi$ satisfying assumptions 2.4 ; as the $y$ dependence of $\Phi$ is not relevant in this subsection we drop explicit reference to it and write $\Phi(x)$. As in the previous subsection we assume that $\mu_{0}(X)=1$. We let $E$ denote the Cameron-Martin space $\operatorname{Im}\left(\mathcal{C}^{\frac{1}{2}}\right)$, equipped with inner product $\left(E,\langle\cdot, \cdot\rangle_{\mathcal{C}}\right)$ and we assume that $m \in E$ so that $\mu_{0}$ is absolutely continuous with respect to the symmetric measure $\mathcal{N}(0, \mathcal{C})[10,17]$. Note that $E$ is compactly embedded in any space which has full measure under $\mu_{0}$, and hence in $X$ [31].

We define probability maximizers to be minimizers of the functional

$$
I(x)=\frac{1}{2}\|x-m\|_{\mathcal{C}}^{2}+\Phi(x)
$$

Such minimizers do indeed maximize the posterior probability density, with respect to Lebesgue measure, in finite dimensions. In infinite dimensions small balls will have highest probability measure when centred on such minimizers. Minimizing $\Phi(x)$ provides the best match to the data; however minimizing sequences $x_{n}$ in $X$ will typically not converge. The quadratic penalization term $\frac{1}{2}\|x-m\|_{\mathcal{C}}^{2}$ forces minimizers to lie in the smaller space $E$ and the key question is whether this penalization, which may be viewed as a generalized form of Tikhonov regularization [66], enables us to extract a minimizer in $E$ from minimizing sequences. This may be viewed as a classical problem in optimal control where $x$ is the control and $I$ is the objective functional, balancing the match to data with regularity constraints. We have the following theorem.

Theorem 2.7. Let assumptions 2.4(i), (ii) hold and assume that $\mu_{0}(X)=1$. Then there exists $\bar{x} \in$ E such that

$$
I(\bar{x})=\bar{I}:=\inf \{I(x): x \in E\} .
$$

Furthermore, if $\left\{x_{n}\right\}$ is a minimizing sequence satisfying $I\left(x_{n}\right) \rightarrow I(\bar{x})$ then there is a subsequence $\left\{x_{n^{\prime}}\right\}$ that converges strongly to $\bar{x}$ in $E$.

Proof. For any $\delta>0$ there is $N=N_{1}(\delta)$ :

$$
\bar{I} \leqslant I\left(x_{n}\right) \leqslant \bar{I}+\delta, \quad \forall n \geqslant N_{1} .
$$

Thus

$$
\frac{1}{2}\left\|x_{n}-m\right\|_{\mathcal{C}}^{2} \leqslant \bar{I}+\delta \quad \forall n \geqslant N_{1} .
$$

Since $m \in E$ the sequence $\left\{x_{n}\right\}$ is bounded in $E$ and, since $E$ is a Hilbert space, there exists $\bar{x} \in E$ such that $x_{n} \rightarrow \bar{x}$ in $E$. By the compact embedding of $E$ in $X$ we deduce that $x_{n} \rightarrow x$, strongly in $X$. By the Lipschitz continuity of $\Phi$ in $X$ we deduce that $\Phi\left(x_{n}\right) \rightarrow \Phi(x)$. Thus $\Phi$ is weakly continuous on $E$.

The functional $J(x):=\frac{1}{2}\|x-m\|_{\mathcal{C}}^{2}$ is weakly lower semicontinuous on $E$. Hence $I(x)=J(x)+\Phi(x)$ is weakly lower semicontinuous on $E$. Since $\Phi$ is bounded from below on $E$ and $m \in E$ we have that $I$ is coercive on $E$. The first result follows from chapter 3, theorem 1.1 , in [16].

To prove the strong convergence of minimizing sequences we generalize an argument from theorem II.2.1 in [46]. By passing to a further subsequence, and for $n, \ell \geqslant N_{2}(\delta)$,

$$
\begin{aligned}
\frac{1}{4}\left\|x_{n}-x_{\ell}\right\|_{\mathcal{C}}^{2} & =\frac{1}{2}\left\|x_{n}-m\right\|_{\mathcal{C}}^{2}+\frac{1}{2}\left\|x_{\ell}-m\right\|_{\mathcal{C}}^{2}-\left\|\frac{1}{2}\left(x_{n}+x_{\ell}\right)-m\right\|_{\mathcal{C}}^{2} \\
& =I\left(x_{n}\right)+I\left(x_{\ell}\right)-2 I\left(\frac{1}{2}\left(x_{n}+x_{\ell}\right)\right)-\Phi\left(x_{n}\right)-\Phi\left(x_{\ell}\right)+2 \Phi\left(\frac{1}{2}\left(x_{n}+x_{\ell}\right)\right) \\
& \leqslant 2(\bar{I}+\delta)-2 \bar{I}-\Phi\left(x_{n}\right)-\Phi\left(x_{\ell}\right)+2 \Phi\left(\frac{1}{2}\left(x_{n}+x_{\ell}\right)\right) \\
& \leqslant 2 \delta-\Phi\left(x_{n}\right)-\Phi\left(x_{\ell}\right)+2 \Phi\left(\frac{1}{2}\left(x_{n}+x_{\ell}\right)\right) .
\end{aligned}
$$


But $x_{n}, x_{\ell}$ and $\frac{1}{2}\left(x_{n}+x_{\ell}\right)$ all converge strongly to $\bar{x}$ in $X$. Thus, by continuity of $\Phi$, we deduce that for all $n, \ell \geqslant N_{3}(\delta)$,

$$
\frac{1}{4}\left\|x_{n}-x_{\ell}\right\|_{\mathcal{C}}^{2} \leqslant 3 \delta \text {. }
$$

Hence the sequence is Cauchy in $E$ and converges strongly and the proof is complete.

\section{Data assimilation in fluid mechanics}

In this section we study four problems involving data assimilation in fluid mechanics. Our aim is to demonstrate that the abstract theory developed in the last section may be applied to concrete problems of interest. We work with simplified model problems in order to exemplify this. We study viscous incompressible fluid flow governed by the Navier-Stokes equation on the two-dimensional unit torus $\mathbb{T}^{2}$ :

$$
\begin{aligned}
& \partial_{t} u-v \Delta u+u \cdot \nabla u+\nabla p=f, \quad \forall(x, t) \in \mathbb{T}^{2} \times(0, \infty) \\
& \nabla \cdot u=0, \quad \forall t \in(0, \infty) \\
& u(x, 0)=u_{0}(x), \quad x \in \mathbb{T}^{2} .
\end{aligned}
$$

We confine ourselves to a two-dimensional torus $\mathbb{T}^{2}$, simply because a mathematical theory of global well-posed solutions in three dimensions has not been found, and to a torus (which is equivalent to a periodic domain) because it removes mathematical technicalities associated with boundaries ${ }^{13}$. In the following we will sometimes find it useful to view $u(\cdot, t)$ as a function of $t$ alone, taking values in a Hilbert space $H$ or $H^{s}$ (with norms $\|\cdot\|,\|\cdot\|_{s}$ defined below) of spatially varying functions; we will then write $u(t)$. We will also sometimes view space-time-dependent functions as taking values in a Hilbert space $\mathcal{H}$ or $\mathcal{H}^{s}$ (with norms $|\cdot|_{0},|\cdot|_{s}$ defined below) of space-time varying functions; we will then write $u$.

In the first two model problems we make noisy observations $y$ which we view as being of the form

$$
y=\mathcal{G}\left(u_{0}\right)+\sigma .
$$

Here $\sigma$ is a mean zero observational noise which, for simplicity, we take to be Gaussian $\mathcal{N}(0, \Sigma)$. Our objective is to determine $u_{0}$, the initial condition for the Navier-Stokes equation, from the finite-dimensional vector of observations $y$. In the second two-model problems, we look at the problem of determining both the initial condition $u_{0}$ and the forcing $f$ from data. Thus we view $y$ as being of the form

$$
y=\mathcal{G}\left(u_{0}, f\right)+\sigma
$$

with $\sigma$ as before. The function $f$ can be viewed as a model error term about which we wish to find information from observed data.

We will consider two kinds of data: Eulerian and Lagrangian. In the former case the data are given as noisy observations of the velocity field at a collection of points in space time. In the second case, the data are given as the positions of a finite collection of Lagrangian trajectories, moving in the velocity field $u$, at a finite set of times. The Lagrangian trajectories are solutions of the equations

$$
\frac{\mathrm{d} z(t)}{\mathrm{d} t}=u(z(t), t), \quad z(0)=z_{0} .
$$
${ }^{13}$ Note that $u_{0}$ or $\left(u_{0}, f\right)$ will play the role of the unknown function $x$ arising in the previous section; this should not
be confused with the use of $x$ to denote the spatial variable in these particular applications to fluid mechanics. 
In the remainder of this section we describe and analyse the observation operator $\mathcal{G}$ in the four different model problems. Our aim is to establish that the theory from the preceding section applies to these problems arising in data assimilation. To do this we need to determine a function space $X$ and prior Gaussian measure $\mu_{0}$ with the properties that the operator $\mathcal{G}: X \rightarrow \mathbb{R}^{m}$ is polynomially bounded and Lipschitz and that $\mu_{0}(X)=1$. We may then apply corollary 2.2, theorems 2.5 and 2.7, since assumptions 2.4 hold for $\Phi$ given by (2.1) if $\mathcal{G}$ is polynomial and Lipschitz. Intuitively our task is to choose the prior measure so that, with probability 1 , functions picked from it are sufficiently regular that the polynomial bounds and Lipschitz properties of the observation operator may be exploited. The key theorems 3.4, 3.8, 3.11 and 3.14 summarize the results. In various lemmas, we also derive further conditions on the observation operator which are useful in the construction, analysis and implementation of variational and MCMC methods on function space-in particular, as well as deriving polynomial bounds and Lipschitz properties of $\Phi$, we also prove differentiability in two cases.

In appendix 1 we describe the mathematical background required to make sense of the equations (3.1)-(3.3) and (3.4) under the desired minimal regularity requirements on the initial condition $u_{0}$ and on the forcing $f$. We also describe the background on Gaussian random fields required to place prior measures on the functions $u_{0}$ and $f$. The reader who wishes to understand all the mathematical details will need to read appendix 1 in its entirety. However a basic understanding of the results in this section may be obtained with the following intuitive interpretations of various operators, norms and discussions of regularity for Gaussian random fields of space or space time.

We start with functions $u: \mathbb{T}^{2} \rightarrow \mathbb{R}^{2}$ which depend on the spatial variable $x$ only. We let $H$ denote the space of square integrable, divergence-free vector fields with average zero over the unit torus. Thus $H$ is a Hilbert space of spatially varying functions only. We denote the norm on $H$ by $\|\cdot\|$. The Stokes operator $A$ may be thought of as the negative Laplacian on $H$. Fractional powers of the Stokes operator can then be defined by diagonalization (Fourier analysis) as would be done for matrices. The Sobolev spaces $H^{s}$ are defined on functions of the spatial variable $x$ alone. In particular $H^{s}$ is the domain of $A^{s / 2}$ and may thus be thought of as the space of divergence-free vector fields with average zero over the unit torus which possess $s$ (possibly fractional) square integrable spatial derivatives. Note that, for every $s>0, H^{s} \subset H$. We denote the norm on $H^{s}$ by $\|\cdot\|_{s}$. On occasion we will also make use of the $L^{p}$ Banach spaces of $x$-dependent functions; in particular the supremum norm over $x$ is denoted by $\|\cdot\|_{L^{\infty}}$.

We now turn to norms on functions of space and time. We let $\mathcal{H}$ denote the Hilbert space of functions $u: \mathbb{T}^{2} \times(0, T) \rightarrow \mathbb{R}^{2}$ with the property that

$$
|u|_{0}^{2}:=\int_{0}^{T}\|u(\cdot, t)\|^{2} \mathrm{~d} t<\infty .
$$

More generally we let $\mathcal{H}^{s}$ denote the Hilbert space of functions $u: \mathbb{T}^{2} \times(0, T) \rightarrow \mathbb{R}^{2}$ with the property that

$$
|u|_{s}^{2}:=\int_{0}^{T}\|u(\cdot, t)\|_{s}^{2} \mathrm{~d} t<\infty .
$$

Thus $\mathcal{H}^{s}=L^{2}\left(0, T ; H^{s}\right)$ and $\mathcal{H}=L^{2}(0, T ; H)$. Note that, for every $s>0, \mathcal{H}^{s} \subset \mathcal{H}$.

When studying problems without model error we will need only to place prior Gaussian random field models on the initial condition $u_{0}$, which is a function of space only. We will work with a particular family of such Gaussian random field models, but before specifying this in general we note that the key idea underlying these prior models is that the variance associated with functions possessing increasing spatial frequency (wave number in a Fourier expansion) should decay sufficiently rapidly under the prior model; this ensures desirable 
regularity properties for functions drawn from the prior. To carry this out in a concrete fashion we employ prior covariance operators which are fractional negative powers of the Stokes operator A. Lemma 4.3 shows how the regularity (number of derivatives) of the Gaussian random field is related to the exponent $\alpha$ in a covariance operator of the form $\beta A^{-\alpha}$. As $\alpha$ grows, the prior variance will decay faster with modulus of the wave number; the parameter $\beta$ can be used to set a scale for the variances. We will typically denote the mean of the initial condition $u_{0}$ by $u_{b}$ to denote the connection with the notation used to denote background state for the initial condition in the applied data assimilation community. Although we work with a particular form of Gaussian prior, the ideas are trivially extended to any Gaussian prior which is absolutely continuous with respect to the measure $\mathcal{N}\left(u_{b}, \beta A^{-\alpha}\right)$. In practical terms this means that asymptotically, for very high wave numbers, the prior measure should have covariances governed by $\beta A^{-\alpha}$; but for any finite wave numbers the practitioner is free to choose the prior measure as he wishes.

When studying problems with model error we will need, in addition, to place prior Gaussian random field models on the forcing $f$ : a function of space and time. Informally this will be in the form of a mean zero statistically stationary solution of a PDE with the form

$$
\frac{\mathrm{d} f}{\mathrm{~d} t}+R f=\eta
$$

where $R$ is a (possibly pseudo) differential operator and $\eta$ is a space-time Gaussian process with correlation operator $\Lambda \delta(t-s)$ for some spatial operator $\Lambda$; thus the noise $\eta$ is white in time and correlated in space with covariance operator $\Lambda$. Full details are given in lemma 4.4. Informally the subsections concerning model error in this section can be understood by recognizing that different choices of $R$ and $\Lambda$ give rise to Gaussian random fields $f$ with different space-time correlations, and different smoothness properties. Roughly speaking smoothness will increase as smoothness of $\Lambda$ increases and as the number of derivatives (possibly fractional) in $R$ increases. Statements such as $D\left(R^{\gamma}\right) \subseteq H^{s}$ mean, roughly, that $R$ is an operator involving $s / \gamma$ (square integrable) derivatives.

\subsection{Eulerian data assimilation}

In weather forecasting, a number of direct observations are made of the air velocity in the atmosphere; these observations can be made from satellites, weather balloons or aircraft, for example. In order to understand how such observations can be combined with a mathematical model, we consider a model problem where the fluid velocity is accurately described by the two-dimensional Navier-Stokes equations (3.1)-(3.3). Suppose we make direct noisy observations of the velocity field at the set of points $\left\{x_{j}\right\}_{j=1}^{J}$ at a set of times $\left\{t_{k}\right\}_{k=1}^{K}$. Then we have $J K$ observations in $\mathbb{R}^{2},\left\{y_{j, k}\right\}_{j, k=1}^{J, K}$. Therefore $m=2 J K$. If $u(x, t)$ is the actual velocity field from which we are taking our noisy observations, then

$$
y_{j, k}=u\left(x_{j}, t_{k}\right)+\sigma_{j, k},
$$

where the $\sigma_{j, k}$ are Gaussian random variables. So in the Euclidean case, our observation operator is constructed from $\left\{u\left(x_{j}, t_{k}\right)\right\}_{j, k=1}^{J, K} \in \mathbb{R}^{2 J K}$, where $u$ is the solution of (3.1)-(3.3). Under suitable regularity conditions on $u_{0}$, the Navier-Stokes equations form a dynamical system, and so $u$ is uniquely defined by $u_{0}$. Hence the observation operator is a function $\mathcal{G}_{E}=\mathcal{G}_{E}\left(u_{0}\right):=\left\{u\left(x_{j}, t_{k}\right)\right\}_{j, k=1}^{J, K}$.

We provide rigorous justification for formula (2.2) in theorem 3.4. The proof relies on the following lemmas which establish a bound on the Eulerian observation operator, and demonstrate that it is Lipschitz. Careful attention is paid to the dependence of the constants on the initial condition $u_{0}$. Proofs of the required estimates on the Navier-Stokes equations 
may be found in appendix 2 . Note that, for the proof of the lemmas, it suffices to consider the case $J=K=1$.

Lemma 3.1. Assume that $u_{0} \in H$ and that $f \in \mathcal{H}^{s}$ for some $s>0$. Then there exists a constant $c$ independent of $u_{0}$ and $f$ such that

$$
\left|\mathcal{G}_{E}\left(u_{0}\right)\right| \leqslant c t_{0}^{-(s+1) / 2}\left(|f|_{s}+|f|_{0}^{2}+\left\|u_{0}\right\|^{2}\right),
$$

provided that $\min _{k} t_{k}>t_{0}>0$.

Proof. Noting by the Sobolev embedding theorem that

$$
\left|u\left(x_{j}, t_{k}\right)\right| \leqslant\left\|u\left(t_{k}\right)\right\|_{L^{\infty}} \leqslant c\left\|u\left(t_{k}\right)\right\|_{1+s}
$$

for any $s>0$, the result follows from the estimate

$$
\|u(t)\|_{1+s}^{2} \leqslant c t^{-(s+1)}\left(|f|_{s}+|f|_{0}^{2}+\left\|u_{0}\right\|^{2}\right)^{2}
$$

obtained in lemma 5.3.

Lemma 3.2. Assume that $u_{0}, v_{0} \in H$ and that $f \in \mathcal{H}$. Then for any $s>0$ there exists a constant $L=L\left(\left\|u_{0}\right\|,\left\|v_{0}\right\|,|f|_{0}\right)$ such that

$$
\left|\mathcal{G}_{E}\left(u_{0}\right)-\mathcal{G}_{E}\left(v_{0}\right)\right| \leqslant L t_{0}^{-(s+1) / 2}\left\|u_{0}-v_{0}\right\|
$$

provided that $\min _{k} t_{k}>t_{0}>0$.

Proof. As above, the result follows from the Sobolev embedding theorem and the estimate on $\|u(t)-v(t)\|_{1+s}$ obtained in lemma 5.5, setting $r=0$.

Lemma 3.3. Let $u_{0} \in H$ and $f \in \mathcal{H}$. Then $\mathcal{G}_{E}\left(u_{0}\right): H \rightarrow \mathbb{R}^{m}$ is differentiable.

Proof. The result is a corollary of differentiability of the solution mapping from $H$ into $H^{1+s}$, using the Sobolev embedding theorem. The proof of lemma 5.6 shows that if $v$ is a second solution of the Navier-Stokes equations with $v(0)=v_{0}$ and $U$ solves

$$
\begin{gathered}
\frac{\mathrm{d} U}{\mathrm{~d} t}+v A U+B(u, U)+B(U, u)=0, \quad \text { with } \quad U(0)=u_{0}-v_{0} \\
\text { then } \theta(t)=u(t)-v(t)-U(t) \text { satisfies, for } t>t_{0}, \\
\|\theta(t)\|_{L^{\infty}} \leqslant\|\theta(t)\|_{1+s} \leqslant t_{0}^{-s} C\left(\left\|u_{0}\right\|,\left\|w_{0}\right\|,|f|_{0}\right)\left\|w_{0}\right\|^{4},
\end{gathered}
$$

which proves the differentiability of $\mathcal{G}_{E}\left(u_{0}\right)$.

Theorem 3.4. Assume that $f \in \mathcal{H}$. Define a Gaussian measure $\mu_{0}$ on $H$, with mean $u_{b} \in H^{\alpha}$ and covariance operator $\beta A^{-\alpha}$ for any $\beta>0, \alpha>1$. Then the probability measure $\mu^{y}\left(d u_{0}\right)=\mathbb{P}\left(d u_{0} \mid y\right)$ is absolutely continuous with respect to $\mu_{0}$ with the Radon-Nikodym derivative

$$
\frac{\mathrm{d} \mu^{y}}{\mathrm{~d} \mu_{0}}\left(u_{0}\right) \propto \exp \left(-\Phi\left(u_{0} ; y\right)\right)
$$

where

$$
\Phi\left(u_{0} ; y\right)=\frac{1}{2}\left|y-\mathcal{G}_{E}\left(u_{0}\right)\right|_{\Sigma}^{2} .
$$

Furthermore, if $f \in \mathcal{H}^{s}$ for some $s>0$ then assumption 2.4 holds for $\Phi$ with $X=H$.

Proof. To establish the first result we use corollary 2.2 with $X=H$. By lemma 4.3, together with the fact that $u_{b}$ is in the Cameron-Martin space for $\mathcal{N}\left(0, A^{-\alpha}\right)$, we have $\mu_{0}(H)=1$. 
Since $\mathcal{G}_{E}(\cdot)$ is continuous on $H$, by lemma 3.2, the first result follows. The second result, namely that assumption 2.4 holds, follows from the polynomial bound of lemma 3.1.

Since we have established that assumption 2.4 holds we deduce that theorems 2.5 and 2.7 apply for any $\alpha>1$. Thus the posterior measure is continuous in the Hellinger metric, and hence expectations of all polynomially bounded functions under the posterior measure, including the mean and covariance operator, are continuous in the data. Furthermore, taking $\alpha=2$, and recalling that $A$ is the Stokes operator, we deduce from theorem 2.7 that the functional

$$
I\left(u_{0}\right)=\frac{1}{2}\left\|A\left(u_{0}-u_{b}\right)\right\|^{2}+\frac{1}{2}\left|y-\mathcal{G}_{E}\left(u_{0}\right)\right|_{\Sigma}^{2}
$$

attains its infimum in $H^{2}$. Furthermore, by lemma $3.3, \mathcal{G}_{E}: H \rightarrow \mathbb{R}$ is differentiable and hence the derivative of $\mathcal{G}_{E}$ is defined in $H^{2}$, making gradient descent and adjoint methods feasible.

\subsection{Lagrangian data assimilation}

Understanding the oceans is fundamental in the atmospheric and environmental sciences, for environmental, commercial and military purposes. One way of probing the oceans is by observing the velocity of the flow at fixed positions in the ocean, which is analogous to the Eulerian data assimilation problem considered in the preceding subsection. Another is to place 'floats' (at a specified depth) or 'drifters' (on the surface) in the ocean and allow them to act as Lagrangian tracers in the flow. These tracers broadcast GPS data concerning their positions and these data can be used to make inference about the oceans themselves.

In order to understand how such observations can be combined with a mathematical model, we again consider a problem where the fluid velocity is accurately described by the two-dimensional Navier-Stokes equations (3.1)-(3.3). We model the Lagrangian instruments which gather data as $J$ tracers $\left\{z_{j}\right\}_{j=1}^{J}$ being transported by the vector field $u$ according to (3.4), which we observe at times $\left\{t_{k}\right\}_{k=1}^{K}$. The tracers' trajectories are then given by the set of ODEs,

$$
z_{j}=u\left(z_{j}, t\right), \quad z_{j}(0)=z_{j, 0},
$$

for some set of starting positions $\left\{z_{j, 0}\right\}_{j=1}^{J}$. Our observations are given by

$$
y_{j, k}=z_{j}\left(t_{k}\right)+\sigma_{j, k} .
$$

Note that each $z_{j}\left(t_{k}\right)$ is a (complicated) function of the initial fluid velocity $u_{0}$, assuming that the $z_{j, 0}$ are known. Thus the observation operator $\mathcal{G}_{L}$ is given by $\mathcal{G}_{L}\left(u_{0}\right)=\left\{z_{j}\left(t_{k}\right)\right\}_{j, k=1}^{J, K}$.

We provide rigorous justification for the formula (2.2) in theorem 3.8, building on the following lemmas which establish a bound on the Lagrangian observation operator, and demonstrate that it is Lipschitz. Again, careful attention is paid to the dependence of the constants on the initial condition $u_{0}$. Again, for the proof of the lemmas, it suffices to consider the case $J=K=1$.

Lemma 3.5. Assume that $u_{0} \in H$ and that $f \in \mathcal{H}$. Then there exists a constant $c$ independent of $u_{0}$ and $f$ such that

$$
\left|\mathcal{G}_{L}\left(u_{0}\right)\right| \leqslant c\left(1+|f|_{0}^{2}+\left\|u_{0}\right\|^{2}\right)^{3 / 2}
$$

Proof. We consider $\mathcal{G}_{L}\left(u_{0}\right)=z(t)$ with $z(t)$ as in (4.1) and $u$ solving (3.1)-(3.3). Since $\|u\|_{L^{\infty}} \leqslant c\|u\|_{1+s}$ for any $s>0$, 


$$
\begin{aligned}
\left|\mathcal{G}_{L}\left(u_{0}\right)\right| & =|z(t)| \leqslant|z(0)|+\int_{0}^{t}\|u(\tau)\|_{L^{\infty}} \mathrm{d} \tau \\
& \leqslant|z(0)|+c \int_{0}^{t}\|u(\tau)\|_{1+s} \mathrm{~d} \tau \\
& \leqslant|z(0)|+c\left(1+|f|_{0}^{2}+\left\|u_{0}\right\|^{2}\right)^{3 / 2},
\end{aligned}
$$

using the bound in (5.10).

Lemma 3.6. Assume that $u_{0}, v_{0} \in H^{s}$ and that $f \in \mathcal{H}^{s}$ for some $s>0$. Then there exists $L=L\left(\left\|u_{0}\right\|_{s},\left\|v_{0}\right\|_{s},|f|_{s}\right)$ such that

$$
\left|\mathcal{G}_{L}\left(u_{0}\right)-\mathcal{G}_{L}\left(v_{0}\right)\right| \leqslant L\left\|u_{0}-v_{0}\right\|_{s} .
$$

Proof. Let $v(t)$ be a solution of the Navier-Stokes equations (3.1) and (3.2) with initial condition $v_{0} \in H^{s}$ (possibly different from $u_{0}$ ) and driving force $g^{14}$.

Let $y(t)$ be the trajectory of the particle initially at $a \in \mathbb{T}^{2}$, moving under the velocity field $v(t)$ :

$$
\frac{\mathrm{d} y}{\mathrm{~d} t}=v(y, t), \quad \text { with } \quad y(0)=a .
$$

When $z(t)$ is the solution of (4.1), the difference $z(t)-y(t)$ satisfies

$$
\begin{aligned}
\frac{\mathrm{d}|z-y|}{\mathrm{d} t} & \leqslant|u(z, t)-v(y, t)| \\
& \leqslant|u(z, t)-u(y, t)|+|u(y, t)-v(y, t)| \\
& \leqslant\|D u\|_{L^{\infty}}|z-y|+\|u-v\|_{L^{\infty}} \\
& \leqslant\|u\|_{2+\epsilon}|z-y|+c\|u-v\|_{1+s},
\end{aligned}
$$

where we choose $0<\epsilon<s$.

Integrating with respect to time we obtain

$$
|z(t)-y(t)| \leqslant c \exp \left(\int_{0}^{t}\|u(\tau)\|_{2+\epsilon} \mathrm{d} \tau\right) \int_{0}^{t}\|u(\tau)-v(\tau)\|_{1+s} \mathrm{~d} \tau .
$$

Since $u_{0} \in H^{s}$ it follows from lemma 5.2 that

$$
\int_{0}^{t}\|u(\tau)\|_{2+\epsilon} \mathrm{d} \tau \leqslant C\left(\left\|u_{0}\right\|_{s},|f|_{\epsilon}\right),
$$

while (5.23) gives bounds on the separation of trajectories in time which imply that

$$
\begin{aligned}
\int_{0}^{t}\|u(\tau)-v(\tau)\|_{1+s} \mathrm{~d} \tau & \leqslant t^{1 / 2}\left(\int_{0}^{t}\|u(\tau)-v(\tau)\|_{1+s}^{2} \mathrm{~d} \tau\right)^{1 / 2} \\
& \leqslant C\left(\left\|u_{0}\right\|_{s},\left\|v_{0}\right\|_{s},|f|_{s}\right)\left\|u_{0}-v_{0}\right\|_{s},
\end{aligned}
$$

from which it follows that

$$
|z(t)-y(t)| \leqslant C\left(\left\|u_{0}\right\|_{s},\left\|v_{0}\right\|_{s},|f|_{s}\right)\left\|u_{0}-v_{0}\right\|_{s}
$$

as required.

Lemma 3.7. Assume that $u_{0} \in H^{s}$ and $f \in \mathcal{H}^{\epsilon}$ for some $\epsilon, s>0$. Then $\mathcal{G}_{L}\left(u_{0}\right): H^{s} \rightarrow \mathbb{R}^{m}$ is differentiable.

${ }^{14}$ Here we take $g$ equal to $f$, but in the analogous proof of lemma 3.13 they are chosen to differ. 
The proof of this lemma is an order of magnitude more involved than those above, and is included in appendix 2, along with the estimates on the Navier-Stokes equations used in all the preceding proofs.

Theorem 3.8. Assume that $f \in \mathcal{H}^{r}$ for some $r>0$. Define a Gaussian measure $\mu_{0}$ on $H$ with mean $u_{b} \in H^{\alpha}$ and covariance operator $\beta A^{-\alpha}$ for any $\beta>0, \alpha>1$. Then the probability measure $\mu^{y}\left(d u_{0}\right)=\mathbb{P}\left(d u_{0} \mid y\right)$ is absolutely continuous with respect to $\mu_{0}$ with the Radon-Nikodym derivative

$$
\frac{\mathrm{d} \mu^{y}}{\mathrm{~d} \mu_{0}}\left(u_{0}\right) \propto \exp \left(-\Phi\left(u_{0} ; y\right)\right),
$$

where

$$
\Phi\left(u_{0} ; y\right)=\frac{1}{2}\left|y-\mathcal{G}_{L}\left(u_{0}\right)\right|_{\Sigma}^{2} .
$$

Furthermore assumption 2.4 holds for $\Phi$ with $X=H^{s}$ for any $s>0$.

The proof is similar to that for theorem 3.4 and we omit it. Similar conclusions to those in the previous section may also be made: the posterior measure is continuous in the Hellinger metric, and hence expectations of all polynomially bounded functions are Lipschitz in the data; and the variational problem

$$
I\left(u_{0}\right)=\frac{1}{2}\left\|A\left(u_{0}-u_{b}\right)\right\|^{2}+\frac{1}{2}\left|y-\mathcal{G}_{L}\left(u_{0}\right)\right|_{\Sigma}^{2}
$$

attains its infimum in $H^{2}$.

\subsection{Eulerian data assimilation with model error}

Data assimilation problems such as that outlined in subsection 3.1 are notoriously hard to solve, especially in situations where there is sensitivity to initial conditions, such as in turbulent fluid flow. In this situation it is desirable to regularize the problem by assuming that the NavierStokes equations are not satisfied exactly, but are subject to a forcing process which can be chosen, in conjunction with the choice of initial condition, to explain the data. The unknown forcing may be viewed as model error.

In order to model this situation we consider the case where the fluid velocity is accurately described by the two-dimensional noisy Navier-Stokes equations (3.1)-(3.3). Suppose we make direct noisy observations of the velocity field at the set of points $\left\{x_{j}\right\}_{j=1}^{J}$ at a set of times $\left\{t_{k}\right\}_{k=1}^{K}$. The observation operator $\mathcal{G}_{\mathrm{EN}}$ is now viewed as a function of both the initial condition $u(x, 0)=u_{0}(x)$ and the driving noise $f: \mathcal{G}_{\mathrm{EN}}=\mathcal{G}_{\mathrm{EN}}\left(u_{0}, f\right)=\left\{u\left(x_{j}, t_{k}\right)\right\}_{j, k=1}^{J, K}$.

We provide rigorous justification for the formula (2.2) in theorem 3.11, building on the following lemmas, whose proofs follow from the estimates in the appendix 2, using arguments identical to those for the case without model error discussed above.

Lemma 3.9. Assume that $u_{0} \in H$ and that $\zeta \in \mathcal{H}^{s}$ for some $s>0$. Then there exists a constant $c$ independent of $u_{0}$ and $\zeta$ such that

$$
\left|\mathcal{G}_{\mathrm{EN}}\left(u_{0}, \zeta\right)\right| \leqslant c t_{0}^{-(s+1) / 2}\left(|\zeta|_{s}+|\zeta|_{0}^{2}+\left\|u_{0}\right\|^{2}\right)
$$

provided that $\min _{k} t_{k}>t_{0}>0$.

Lemma 3.10. Assume that $u_{0}, v_{0} \in H$ and that $\zeta, \xi \in \mathcal{H}^{s}$ for some $s>0$. Then there exists $L=L\left(\left\|u_{0}\right\|,\left\|v_{0}\right\|,|\zeta|_{0},|\xi|_{0}, s\right)$ such that

$$
\left|\mathcal{G}_{\mathrm{EN}}\left(u_{0}, \zeta\right)-\mathcal{G}_{\mathrm{EN}}\left(v_{0}, \xi\right)\right| \leqslant L t_{0}^{-(s+1) / 2}\left(\left\|u_{0}-v_{0}\right\|^{2}+|\zeta-\xi|_{s}^{2}\right)^{\frac{1}{2}},
$$


provided that $\min _{k} t_{k}>t_{0}>0$.

Theorem 3.11. Assume that, for some $s>0$ and $\gamma \in\left(0, \frac{1}{2}\right], D\left(R^{\gamma}\right) \subseteq H^{s}$. Define a Gaussian measure $\mu_{0}$ on $H \times \mathcal{H}$ by choosing $u_{0}$ distributed according to the Gaussian measure on $H$ with mean $u_{b}$ and covariance operator $\beta A^{-\alpha}$, any $\beta>0, \alpha>1$, and $f$ independent of $u_{0}$ and distributed according to stationary measure $\nu_{0}$ on $\mathcal{H}$ given by equation (3.5) (constructed in lemma 4.4). If $u_{b} \in H^{\alpha}$ then the probability measure $\mu^{y}\left(\mathrm{~d} u_{0}, \mathrm{~d} f\right)=\mathbb{P}\left(\mathrm{d} u_{0}, \mathrm{~d} f \mid y\right)$ is absolutely continuous with respect to $\mu_{0}$ with the Radon-Nikodym derivative

$$
\frac{\mathrm{d} \mu^{y}}{\mathrm{~d} \mu_{0}}\left(u_{0}, f\right) \propto \exp \left(-\Phi\left(u_{0}, f ; y\right)\right)
$$

where

$$
\Phi\left(u_{0}, f ; y\right)=\frac{1}{2}\left|y-\mathcal{G}_{\mathrm{EN}}\left(u_{0}, f\right)\right|_{\Sigma}^{2} .
$$

Furthermore, assumption 2.4 holds for $\Phi$ with $X=H \times \mathcal{H}^{s}$ for any $s>0$.

Proof. To establish the first result we use corollary 2.2 with $X=H \times \mathcal{H}^{s}$ for some $s>0$ sufficiently small. By lemmas 4.3 and 4.4 together with the fact that $u_{b}$ is in the CameronMartin space for $\mathcal{N}\left(0, A^{-\alpha}\right)$ and the assumptions on $D\left(R^{\gamma}\right)$ we have $\mu_{0}(X)=1$. Since $\mathcal{G}_{\mathrm{EN}}(\cdot)$ is continuous on $X$, by lemma 3.10 , the first result follows. The second result, namely that assumption 2.4 holds, follows from the polynomial bound of lemma 3.9.

Thus the posterior measure on $\left(u_{0}, f\right)$ is Lipschitz continuous, with respect to changes in data, in the Hellinger metric, and hence expectations of polynomially growing functions of $\left(u_{0}, f\right)$ perturb smoothly with respect to changes in the data.

We can also study variational problems. To do this we need to identify the CameronMartin space for the Gaussian reference measure $\mu_{0}$. For the posterior measure to be well defined we choose the measure on the initial condition to have covariance operator $A^{-\alpha}$, for any $\alpha>1$; for the prior on the forcing we choose $R$ so that, for some $s>0, D\left(R^{\frac{1}{2}}\right) \subseteq H^{s}$. The Cameron-Martin space $E$ is the subspace of $H \times \mathcal{H}$ determined by the space-time inner product $\langle\cdot, \cdot\rangle_{E}$ which we now define. In the following note that $u_{i}$ are initial conditions and functions of space only; the $f_{i}$ are forcing functions of space and time. With this in hand we may define

$$
\begin{aligned}
\left\langle\left(u_{1}, f_{1}\right),\left(u_{2}, f_{2}\right)\right\rangle_{E}= & \left(A^{\alpha / 2} u_{1}, A^{\alpha / 2} u_{2}\right) \\
& +\left(\left\langle f_{1}(0), f_{2}(0)\right\rangle_{R^{-1} \Lambda}+\left\langle f_{1}(T), f_{2}(T)\right\rangle_{R^{-1} \Lambda}\right) \\
& +\int_{0}^{T}\left(\left\langle\frac{\mathrm{d} f_{1}}{\mathrm{~d} t}(t), \frac{\mathrm{d} f_{2}}{\mathrm{~d} t}(t)\right\rangle_{\Lambda}+\left\langle R f_{1}(t), R f_{2}(t)\right\rangle_{\Lambda}\right) \mathrm{d} t .
\end{aligned}
$$

The norm is thus

$$
\begin{aligned}
\left\|\left(u_{0}, f\right)\right\|_{E}^{2}= & \left\|A^{\alpha / 2} u_{0}\right\|^{2}+\left(\|f(0)\|_{R^{-1} \Lambda}^{2}+\|f(T)\|_{R^{-1} \Lambda}^{2}\right) \\
& +\int_{0}^{T}\left(\left\|\frac{\mathrm{d} f}{\mathrm{~d} t}(t)\right\|_{\Lambda}^{2}+\|R f(t)\|_{\Lambda}^{2}\right) \mathrm{d} t .
\end{aligned}
$$

Thus $E$ is contained in $H^{\alpha} \times E^{\prime}$ where

$$
E^{\prime}=H^{1}\left(0, T ; D\left(\Lambda^{-\frac{1}{2}}\right)\right) \cap L^{2}\left(0, T ; D\left(\Lambda^{-\frac{1}{2}} R\right)\right) .
$$

By lemmas 3.9 and 3.10, the function $\Phi$ is continuous from $X=H \times \mathcal{H}^{s}$ for any $s>0$. Since $\Lambda$ is trace class we have

$$
E^{\prime} \subseteq H^{1}(0, T ; H) \cap L^{2}(0, T ; D(R)) .
$$


Hence by the compactness theorem 8.1 in [62] we deduce that $E^{\prime}$ is compactly embedded into $L^{2}\left(0, T ; D\left(R^{\frac{1}{2}}\right)\right)$. Since $H^{\alpha}$ is compactly embedded into $H$, it is immediate that $H^{\alpha} \times E^{\prime}$ is compactly embedded into $H \times L^{2}\left(0, T ; D\left(R^{\frac{1}{2}}\right)\right)$ and hence into $X=H \times \mathcal{H}^{s}$. Theorem 2.7 then shows that the functional

$$
I\left(u_{0}, f\right)=\frac{1}{2}\left\|\left(u_{0}, f\right)\right\|_{E}^{2}+\frac{1}{2}\left|y-\mathcal{G}_{\mathrm{EN}}\left(u_{0}, f\right)\right|_{\Sigma}^{2}
$$

attains its infimum in $E$.

\subsection{Lagrangian data assimilation with model error}

In the case of Lagrangian data, the problem can exhibit sensitivity to initial conditions even when the underlying fluid flow is not itself chaotic. This is since the particle trajectories can still exhibit chaoic behaviour in this case. It is again of interest in this situation to assume that the equations of fluid motion are subject to noise. In order to understand this situation we again consider the case where the fluid velocity is accurately described by the twodimensional Navier-Stokes equations (3.1)-(3.3). We again model the Lagrangian instruments which gather data as $J$ tracers $\left\{z_{j}\right\}_{j=1}^{J}$ being transported by the vector field $u$ according to equation (3.8), which we observe at times $\left\{t_{k}\right\}_{k=1}^{K}$. Since $u$ in (3.8) is uniquely determined by $u_{0}$ and $f$, the observation operator is given by $\mathcal{G}_{\mathrm{LN}}=\mathcal{G}_{\mathrm{LN}}\left(u_{0}, f\right)=\left\{z_{j}\left(t_{k}\right)\right\}_{j, k=1}^{J, K}$.

We provide rigorous justification for the formula (2.2) in theorem 3.14. The results follow from the next lemmas which provide the requisite bounds and Lipschitz properties on the observation operator. Again, the proofs of the following lemmas follow the same arguments as those without model error discussed in detail above.

Lemma 3.12. Assume that $u_{0} \in H$ and that $\zeta \in \mathcal{H}$. Then there exists a constant $c$ independent of $u_{0}$ and $\zeta$ such that

$$
\left|\mathcal{G}_{\mathrm{LN}}\left(u_{0}, \zeta\right)\right| \leqslant c\left(1+|\zeta|_{0}^{2}+\left\|u_{0}\right\|^{2}\right)^{3 / 2}
$$

Lemma 3.13. Assume that $u_{0}, v_{0} \in H^{s}$ and that $\zeta, \xi \in \mathcal{H}^{s}$ for some $s>0$. Then there exists $L=L\left(\left\|u_{0}\right\|_{s},\left\|v_{0}\right\|_{s},|\zeta|_{s},|\xi|_{s}\right)$ such that

$$
\left|\mathcal{G}_{L}\left(u_{0}, \zeta\right)-\mathcal{G}_{L}\left(v_{0}, \xi\right)\right| \leqslant L\left(\left\|u_{0}-v_{0}\right\|_{s}^{2}+|\zeta-\xi|_{0}^{2}\right)^{\frac{1}{2}}
$$

Theorem 3.14. Assume that, for some $s>0$ and $\gamma \in\left(0, \frac{1}{2}\right], D\left(R^{\gamma}\right) \subseteq H^{s}$. Define a Gaussian measure $\mu_{0}$ on $H \times \mathcal{H}$ by choosing $u_{0}$ distributed according to the Gaussian measure on $H$ with mean $u_{b} \in H^{\alpha}$ and covariance operator $\beta A^{-\alpha}$, any $\beta>0, \alpha>1$, and $f$ independent of $v$ and distributed according to the stationary measure $v_{0}$ on $\mathcal{H}$ constructed in lemma 4.4. If $u_{b} \in H^{\alpha}$ then the probability measure $\mu^{y}\left(\mathrm{~d} u_{0}, \mathrm{~d} f\right)=\mathbb{P}\left(\mathrm{d} u_{0}, \mathrm{~d} f \mid y\right)$ is absolutely continuous with respect to $\mu_{0}$ with the Radon-Nikodym derivative

$$
\frac{\mathrm{d} \mu^{y}}{\mathrm{~d} \mu_{0}}\left(u_{0}, f\right) \propto \exp \left(-\Phi\left(u_{0}, f ; y\right)\right),
$$

where

$$
\Phi\left(u_{0}, f ; y\right)=\frac{1}{2}\left|y-\mathcal{G}_{\mathrm{LN}}\left(u_{0}, f\right)\right|_{\Sigma}^{2}
$$

Furthermore, assumption 2.4 holds with $X=H^{s} \times \mathcal{H}^{s}$ for any $s>0$ sufficiently small.

The situation here is identical to that in the previous subsection, but taking $X=H^{s} \times \mathcal{H}^{s}$ for some $s>0$ sufficiently small; we omit the proof of the theorem. We again have continuity 
of expectations of all polynomially bounded functions of $\left(u_{0}, f\right)$. The Cameron-Martin space is the same as in the previous subsection and the functional $I\left(u_{0}, f\right)$ given by

$$
I\left(u_{0}, f\right)=\frac{1}{2}\left\|\left(u_{0}, f\right)\right\|_{E}^{2}+\frac{1}{2}\left|y-\mathcal{G}_{\mathrm{LN}}\left(u_{0}, f\right)\right|_{\Sigma}^{2}
$$

attains its infimum in $E$.

\section{Appendix 1: Mathematical setting for applications to fluid mechanics}

We describe the mathematical setting for the differential equations of interest to us when applying the theory of section 2 to applications arising in fluid mechanics described in section 3 . We also include discussion of the requisite theory of Gaussian measures in Hilbert space, including linear stochastic PDEs, required to define prior measures on the initial condition (a measure on functions of space only) and on the model error (a measure on functions of space time).

\subsection{Navier-stokes equations and the stokes operator}

We study viscous incompressible fluid flow governed by the Navier-Stokes equation on the two-dimensional unit torus $\mathbb{T}^{2}$ given by equations (3.1)-(3.3). The following result from [67] shows existence and uniqueness of weak solutions under these assumptions. Let $L^{2}$ denote the space of square integrable functions on $\mathbb{T}^{2}$. Define the Hilbert space

$$
H=\left\{u \in L^{2}, \int_{\mathbb{T}^{2}} u \mathrm{~d} x=0, \nabla \cdot u=0\right\},
$$

with inner product denoted by $(f, g):=\int_{\mathbb{T}^{2}} f(x) g(x) \mathrm{d} x$, and the resulting norm $\|\cdot\|$. We define $H^{s}$ to be the Sobolev space of functions on $\mathbb{T}^{2}$ with $s$ weak derivatives in $L^{2}$; for the moment $s$ is an integer but we extend to non-integer $s$ below. We define $V=H^{1} \bigcap H$ and denote the dual space of $V$ by $V^{\prime}$.

Theorem 4.1. [67] Assume that $u_{0} \in H$ and $f \in L^{2}\left(0, T ; V^{\prime}\right)$. Then there exists a unique weak solution $u$ to the Navier-Stokes equations (3.1)-(3.3) satisfying

$$
u \in L^{2}(0, T ; V) \cap L^{\infty}(0, T ; H) .
$$

In the following we use $\langle\cdot, \cdot\rangle$ to denote the inner product on $\mathbb{R}^{m}$ or $\mathbb{C}^{m}$, and $|\cdot|$ denotes the resulting norm. We let $P$ denote the orthogonal projection from $L^{2}$ into $H$ (the Leray projector) and define the Stokes operator by $A u=-P \triangle u$ for all $u \in D(A)$, where

$$
D(A)=\{u \in H, \triangle u \in H\}=H^{2} \cap H .
$$

We can create an orthonormal basis for $H$ by letting

$$
\phi_{k}=\frac{k^{\perp}}{|k|} \exp (2 \pi \mathrm{i} k . x)
$$

where $k=\left(k_{1}, k_{2}\right)$ and $k^{\perp}=\left(k_{2},-k_{1}\right)$; note that $\nabla \cdot \phi_{k}=0$ so that the basis is divergence free. We define $u_{k}=\left\langle u, \phi_{k}\right\rangle$. Then any $u \in H$ may be written as

$$
u=\sum_{k} u_{k} \phi_{k}
$$

Here, and in what follows, all sums in $k$ are over $\mathbb{Z}^{2} \backslash\{0\}$. The function $u$ is real and so $\bar{u}_{-k}=u_{k}$. Note that $A$ acts as the negative Laplacian on its domain, the $\phi_{k}$ are its eigenfunctions, and $A \phi_{k}=4 \pi^{2}|k|^{2} \phi_{k}$. We denote the eigenvalues $a_{k}=4 \pi^{2}|k|^{2}$. In this setting we can specify the 
Sobolev spaces $H^{s}$ by $H^{s}=\left\{u \in H: \sum_{k} a_{k}^{s}\left|u_{k}\right|^{2}<\infty\right\}$, with norm $\|u\|_{s}=\left(\sum_{k} a_{k}^{s}\left|u_{k}\right|^{2}\right)^{1 / 2}$. This agrees with the preceding definition in the case where $s$ is an integer, but is valid for any $s \in \mathbb{R}$ and hence gives notions of spaces of functions with fractional and negative derivatives. Note that $\|\cdot\|_{0}=\|\cdot\|$ and that $\|\cdot\|_{1}$ is a norm on $V$.

The Navier-Stokes equation is sometimes written as an ODE in the Hilbert space $H$ and we use this compact notation:

$$
\frac{\mathrm{d} u}{\mathrm{~d} t}+v A u+B(u, u)=f, \quad u(0)=u_{0} .
$$

Here $B(u, u)$ is the bilinear form found from projecting the nonlinear term $u \cdot \nabla u$ into $H$, and (abusing notation) $f$ is now the original forcing, projected into $H$.

\subsection{Lagrangian trajectories}

Given a velocity field $u(x, t)$ from the Navier-Stokes equation, it is natural to wish to define Lagrangian trajectories describing the motion of fluid particles advected by the flow. Doing so is integral to the mathematical formulation of Lagrangian data assimilation. Our goal is thus to make sense of the ordinary differential equation (3.4) in the case where the initial velocity field $u(\cdot, 0)=u_{0}$ may not be regular enough to employ the classical theory of differential equations. Thus we consider the integral equation formulation of the trajectory equations, namely

$$
z(t)=z_{0}+\int_{0}^{t} u(z(s), s) \mathrm{d} s, z(0)=z_{0} .
$$

The following result from $[12,18]$ achieves such a result with minimal regularity assumptions on the initial condition for the velocity field.

Theorem 4.2. $[12,18]$ For $u_{0} \in H$ and $f \in L^{2}(0, T ; H)$, let $u \in L^{2}(0, T ; V) \cap L^{\infty}(0, T ; H)$ be a weak solution of the two dimensional Navier-Stokes equation (3.1-3.3), extended by periodicity to a function $u: \mathbb{R}^{2} \times(0, T] \rightarrow \mathbb{R}^{2}$. Then the ordinary differential equation (4.1) has a unique solution $z \in C\left(\mathbb{R}^{+}, \mathbb{R}^{2}\right)$.

\subsection{Gaussian measures on Hilbert space}

We setup the machinery to define prior measures on the initial condition and on the driving force for the Navier-Stokes equation. We will assume that both prior measures are Gaussian.

We will define a prior distribution for the initial condition $u_{0}$ to be the Gaussian measure $\mu_{0}=\mathcal{N}\left(u_{b}, A^{-\alpha}\right)$, where $\alpha>1$ to ensure that the covariance operator is trace class. To make a sample from this distribution, we can use the Karhunen-Loève expansion: if

$$
u=u_{b}+\sum_{k} a_{k}^{-\alpha / 2} \phi_{k} \xi_{k},
$$

where $\xi_{k}$ is a sequence of complex unit Gaussians, i.i.d except for the reality constraint that $\bar{\xi}_{-k}=\xi_{k}$, then $u \sim \mathcal{N}\left(u_{b}, A^{-\alpha}\right)$. We now wish to ascertain the values of $\alpha$ for which $\left(u-u_{b}\right) \in H^{s}$. The proof technique used for the following lemma is well known and may be found in, for example, [17].

Lemma 4.3. If $\alpha>s+1$ then $\left(u-u_{b}\right) \in H^{s}, \mu_{0}$-almost surely. 
Proof. It is enough to show that $\mathbb{E}\left(\left\|u-u_{b}\right\|_{s}^{2}\right)<\infty$ for $s<\alpha-1$.

$$
\begin{aligned}
\mathbb{E}\left(\left\|u-u_{b}\right\|_{s}^{2}\right) & =\mathbb{E}\left(\sum_{k} a_{k}^{s}\left|a_{k}^{-\alpha / 2} \xi_{k}\right|^{2}\right) \\
& =\sum_{k} a_{k}^{s-\alpha} \mathbb{E}\left|\xi_{k}\right|^{2} \\
& \propto \sum_{k}|k|^{2(s-\alpha)} .
\end{aligned}
$$

Since we are in two dimensions, this sum is finite if $s<\alpha-1$, as may be shown by comparison with an integral.

For the prior on the model error (driving noise), we will employ Gaussian measures on the Hilbert space $\mathcal{H}=L^{2}(0, T ; H)$. We will construct these measures through stationary solutions of an infinite-dimensional Ornstien-Uhlenbeck (OU) process. We write this as an SDE in Hilbert space $H$ :

$$
\frac{\mathrm{d} f}{\mathrm{~d} t}+R f=\sqrt{\Lambda} \frac{\mathrm{d} W}{\mathrm{~d} t} .
$$

Here $W$ is cylindrical Brownian motion in $H$ :

$$
W(t)=\sum_{k} \phi_{k} \beta_{k}(t)
$$

where the $\beta_{k}$ are independent standard complex valued Brownian motions, except for the correlation arising from imposing the reality constraint $\phi_{-k}=\bar{\phi}_{k}$; thus $\mathrm{d} W / \mathrm{d} t$ is white in time (because the derivative of Brownian motion is white) and white in space (because all Fourier components are statistically identical). We assume that $R$ and $\Lambda$ are self-adjoint positive operators in $H$, diagonalized in the same basis as the Stokes operator $A$. Thus

$$
R \phi_{k}=r_{k} \phi_{k}, \quad \Lambda \phi_{k}=\lambda_{k} \phi_{k} .
$$

The $\left\{r_{k}\right\}$ and $\left\{\lambda_{k}\right\}$ are positive. We assume that $R$ generates an analytic semigroup, so that $\exp (-R t)$ maps bounded functions into analytic ones, and that $\Lambda$ is trace-class so that its eigenvalues are summable. To understand (4.2), note that each component of $f$ in the basis $\left\{\phi_{k}\right\}$ is a (complex) scalar-valued OU process. As such, each component is ergodic: the statistical distribution converges exponentially fast to a Gaussian in time; furthermore, this Gaussian distribution is preserved by the OU process, giving rise to a stationary Gaussian process with exponential correlations. Synthesizing the behaviour in each individual Fourier component we deduce that $f$ is itself ergodic, and is stationary if given an appropriate Gaussian distribution in $H$. This, together with a regularity statement, is the content of the following lemma.

Lemma 4.4. Equation (4.2) is ergodic with invariant measure $\mathcal{N}\left(0, \frac{1}{2} R^{-1} \Lambda\right)$. If $f(0) \sim$ $\mathcal{N}\left(0, \frac{1}{2} R^{-1} \Lambda\right)$, independently of $W$, then $f(t)$ is stationary with $f(t) \sim \mathcal{N}\left(0, \frac{1}{2} R^{-1} \Lambda\right)$ for all $t>0$ and, almost surely with respect to $f(0)$ and $W, f \in L^{2}\left(0, \infty ; D\left(R^{\gamma}\right)\right)$ for all $\gamma \in\left(0, \frac{1}{2}\right]$.

Proof. Ergodicity follows from theorems 11.7 and 11.11 in [17]. The stationary solution is given by

$$
W_{R}(t):=\int_{-\infty}^{t} \exp (-R(t-s)) \sqrt{\Lambda} \mathrm{d} W(s)
$$


By use of the Karhunen-Loève expansion we have

$$
f(0)=\sum_{k} \sqrt{\frac{\lambda_{k}}{2 r_{k}}} \phi_{k} \xi_{k}
$$

with $\left\{\xi_{k}\right\}$ a sequence of complex unit Gaussians, i.i.d. except for the reality constraint that $\bar{\xi}_{-k}=\xi_{k}$. Then for $\gamma \in\left(0, \frac{1}{2}\right]$, since $\Lambda$ is trace class,

$$
\begin{aligned}
\mathbb{E}\left\|R^{\gamma} f(0)\right\|^{2} & =\sum_{k} \frac{1}{2} r_{k}^{2 \gamma-1} \lambda_{k} \\
& \leqslant c \sum_{k} \frac{\lambda_{k}}{2} \\
& <\infty .
\end{aligned}
$$

By stationarity

$$
\mathbb{E}\left\|R^{\gamma} f(t)\right\|^{2}=\mathbb{E}\left\|R^{\gamma} f(0)\right\|^{2} .
$$

Thus

$$
\mathbb{E} \int_{0}^{T}\left\|R^{\gamma} f(t)\right\|^{2} \mathrm{~d} t=T \mathbb{E}\left\|R^{\gamma} f(0)\right\|^{2} .
$$

Since the right-hand side is finite for $\gamma \in\left(0, \frac{1}{2}\right]$ we deduce that $f \in L^{2}\left(0, T ; D\left(R^{\gamma}\right)\right)$ for $\gamma \in\left(0, \frac{1}{2}\right]$, almost surely.

\subsection{The noisy Navier-stokes equations}

We wish to make sense of the Navier-Stokes equation driven by a noise process $f$ :

$$
\begin{aligned}
& \partial_{t} u-v \triangle u+u \cdot \nabla u+\nabla p=f, \quad \forall(x, t) \in \mathbb{T}^{2} \times(0, \infty) \\
& \nabla \cdot u=0, \quad \forall t \in(0, \infty) \\
& u(x, 0)=u_{0}(x), \quad x \in \mathbb{T}^{2} .
\end{aligned}
$$

We assume that $f$ is a mean zero Gaussian process. Specifically we will be interested in the case where $f$ is given by the OU process solving (4.2). As a pair of differential equations in $H \times H$ we have ${ }^{15}$

$$
\begin{aligned}
& \frac{\mathrm{d} u}{\mathrm{~d} t}+A u+B(u, u)=f, \quad u(0)=u_{0}, \\
& \frac{\mathrm{d} f}{\mathrm{~d} t}+R f=\sqrt{\Lambda} \frac{\mathrm{d} W}{\mathrm{~d} t}, \quad f(0) \sim \mathcal{N}\left(0, \frac{1}{2} R^{-1} \Lambda\right) .
\end{aligned}
$$

We let

$$
|\psi|_{0}^{2}=\int_{0}^{T}\|\psi(t)\|^{2} \mathrm{~d} t \quad \text { and } \quad|\psi|_{l}^{2}=\int_{0}^{T}\|\psi(t)\|_{l}^{2} \mathrm{~d} t
$$

denote the norms in $\mathcal{H}:=L^{2}(0, T ; H)$ and $\mathcal{H}^{l}:=L^{2}\left(0, T ; H^{l}\right)$, respectively.

We assume that $R$ is such that, for some $s \geqslant 0$ and $\gamma \in\left(0, \frac{1}{2}\right], D\left(R^{\gamma}\right) \subseteq H^{s}$. Then, by lemma 4.4 , the law of the stationary paths $f(t)$ given by (4.2) is a probability measure

${ }^{15}$ Again we abuse notation and write $f$ for $P f$. 
which has full measure on $\mathcal{H}^{s}$; as such we view it as a probability measure on this space, by considering an appropriate version. We denote this measure by $v_{0}$. A straightforward application of theorem 4.1 gives the following result:

Theorem 4.3. Assume that $R$ is such that, for some $s \geqslant 0$ and $\gamma \in\left(0, \frac{1}{2}\right], D\left(R^{\gamma}\right) \subseteq H^{s}$. Let $f$ be distributed according to the measure $v_{0}$ given by a stationary solution of (4.2). Then, for $u_{0} \in H$, (4.3)-(4.5) has a weak solution $u \in L^{2}(0, T ; V) \cap L^{\infty}(0, T ; H)$, v $v_{0}$ almost surely.

\section{Appendix 2: Estimates on solutions of the Navier-Stokes equations}

This appendix contains detailed estimates on solutions, and differences of solutions, of the Navier-Stokes equations. Many of the basic estimates and tools in this section derive from using modifications of techniques frequently used in the analysis of the Navier-Stokes equations (see [62], for example). However, our emphasis is on isolating precisely the dependence on initial data and forcing and hence it is not possible to simply quote existing results. Throughout, lower case $c$ is a constant, independent of initial condition and driving force, but depending on $v$ and possibly changing between occurrences. We first derive a number of results concerning solutions of the Navier-Stokes equations, or differences of solutions of the equations, all of which are used in proving the lemmas in this section. The results assume either $u_{0} \in H$, required for the Eulerian case, or $u_{0} \in H^{s}$ for $s>0$, required in the Lagrangian case.

In what follows we use the notation

$$
D^{m}=\sum_{\alpha_{1}+\alpha_{2}=m} \frac{\partial^{\alpha_{1}+\alpha_{2}}}{\partial x_{1}^{\alpha_{1}} \partial x_{2}^{\alpha_{2}}}
$$

with $\alpha_{1}, \alpha_{2} \in \mathbb{N}$ and $\left(x_{1}, x_{2}\right) \in \mathbb{T}^{2}$.

\subsection{Orthogonality relations and bounds for the nonlinear term}

In this section, we will frequently have to find upper bounds on expressions involving the nonlinear term of the form $\left|\left((u \cdot \nabla) v, A^{s} w\right)\right|$, so we will give a short derivation of these estimates here. However, before deriving bounds on such expressions, we note that

$$
((u \cdot \nabla) v, v)=0
$$

which shows in particular that the nonlinear term makes no contribution to the kinetic energy. (This equality is easy to check using an integration by parts.) Since we are working in a two-dimensional periodic domain, we also have the higher order orthogonality relation

$$
((u \cdot \nabla u), A u)=0,
$$

which can be checked by expanding in components and using the divergence-free property to cancel appropriately grouped terms.

The following bounds on the trilinear form $((u \cdot \nabla) v, w)$ will be used repeatedly in what follows.

Lemma 5.1. For $s=0$,

$$
|((u \cdot \nabla) v, w)| \leqslant c\|u\|^{1 / 2}\|u\|_{1}^{1 / 2}\|v\|_{1}\|w\|^{1 / 2}\|w\|_{1}^{1 / 2} .
$$

For $0<s<1$,

$$
\left|\left((u \cdot \nabla) v, A^{s} w\right)\right| \leqslant c\|u\|_{s}\|v\|_{1}\|w\|_{1+s} .
$$


For $0<s \leqslant 1$

$$
\left|\left((u \cdot \nabla) v, A^{s} w\right)\right| \leqslant c\|u\|^{1 / 2}\|u\|_{s}^{1 / 2}\|v\|_{1}^{1 / 2}\|v\|_{s+1}^{1 / 2}\|w\|_{s+1} .
$$

For $s=1+l, 0<l<1$,

$$
\left|\left((u \cdot \nabla) v, A^{1+l} w\right)\right| \leqslant c\|u\|_{1+l}\|v\|_{1}\|w\|_{2+l}+c\|u\|_{l}\|v\|_{2}\|w\|_{2+l} .
$$

Proof. For $s=0$ write

$$
\begin{aligned}
|((u \cdot \nabla) v, w)| & \leqslant\||u\|D v\| A w|\|_{L^{1}} \\
& \leqslant\|u\|_{L^{4}}\|D v\|_{L^{2}}\|w\|_{L^{4}} \quad \text { (Hölder's inequality) } \\
& \leqslant c\|u\|^{1 / 2}\|u\|_{1}^{1 / 2}\|v\|_{1}\|w\|^{1 / 2}\|w\|_{1}^{1 / 2},
\end{aligned}
$$

where the last line follows using Ladyzhenskaya's inequality

$$
\|u\|_{L^{4}} \leqslant c\|u\|^{1 / 2}\|u\|_{1}^{1 / 2} \text {. }
$$

For $0<s<1$ we have

$$
\begin{aligned}
\left|\left((u \cdot \nabla) v, A^{s} w\right)\right| & \leqslant\left\|\left|u\|D v\| A^{s} w\right|\right\|_{L^{1}} \\
& \leqslant\|u\|_{L^{2 /(1-s)}}\|D v\|_{L^{2}}\left\|A^{s} w\right\|_{L^{2 / s}} \quad \text { (Hölder's inequality) } \\
& \leqslant c\|u\|_{s}\|v\|_{1}\left\|A^{s} w\right\|_{1-s} \quad \text { (Sobolev embedding) } \\
& \leqslant c\|u\|_{s}\|v\|_{1}\|w\|_{1+s},
\end{aligned}
$$

where the Sobolev embedding result used is the inclusion $L^{r} \subset H^{1-(2 / r)}$ valid for twodimensional domains. Using different $L^{p}$ spaces in Hölder's inequality we can obtain

$$
\begin{aligned}
& \left|\left((u \cdot \nabla) v, A^{s} w\right)\right| \leqslant\left\|| u | \left|D v\left\|A^{s} w \mid\right\|_{L^{1}}\right.\right. \\
& \leqslant\|u\|_{L^{4 / 2-s)}}\|D v\|_{L^{4 /(2-s)}}\left\|A^{s} w\right\|_{L^{2 / s}} \quad \text { (Hölder's inequality) } \\
& \leqslant\|u\|_{L^{2}}^{1 / 2}\|u\|_{L^{2 /(1-s)}}^{1 / 2}\|D v\|_{L^{2}}^{1 / 2}\|D v\|_{L^{2 /(1-s)}}^{1 / 2}\left\|A^{s} w\right\|_{L^{2 / s}} \quad\left(L^{p} \text { interpolation }\right) \\
& \leqslant c\|u\|^{1 / 2}\|u\|_{s}^{1 / 2}\|v\|_{1}^{1 / 2}\|v\|_{s+1}^{1 / 2}\|w\|_{s+1} \quad \text { (Sobolev embedding); }
\end{aligned}
$$

a result that is also true for $s=1$ using (5.7) in place of the $L^{p}$ interpolation and Sobolev embedding results.

For $s=1+l$

$$
\begin{aligned}
\left((u \cdot \nabla) v, A^{1+l} w\right)= & \sum_{k=1}^{2}\left((u \cdot \nabla) v,-\frac{\partial^{2}}{\partial x_{k}^{2}} A^{l} w\right) \\
= & \sum_{k=1}^{2}\left(\left(\frac{\partial}{\partial x_{k}} u \cdot \nabla\right) v+\left(u \cdot \frac{\partial}{\partial x_{k}} \nabla\right) v, \frac{\partial}{\partial x_{k}} A^{l} w\right) \quad \text { (parts) } \\
\leqslant & 2\left\|\left|D u\|D v\| D A^{l} w\right|\right\|_{L^{1}}+2\|\mid u\| D^{2} v\left\|D A^{l} w\right\|_{L^{1}} \\
\leqslant & c\|D u\|_{L^{2 / 1-l}}\|D v\|\left\|D A^{l} w\right\|_{L^{2 / l}} \\
& +c\|u\|_{L^{2 /(1-l)}}\left\|D^{2} v\right\|\left\|D A^{l} w\right\|_{L^{2 / l}} \quad \text { (Hölder) } \\
\leqslant & c\|u\|_{1+l}\|v\|_{1}\|w\|_{2+l}+c\|u\|_{l}\|v\|_{2}\|w\|_{2+l} \text {. (Sobolev) }
\end{aligned}
$$

\subsection{Bounds on solutions}

Here we give bounds on the norms of solutions, and on integrals of those norms. Since often bounds on quantities like $\|u\|_{s}$ will depend on integral bounds on the same (or similar) quantities, we will adopt the notation

$$
K_{s}(t)=\int_{0}^{t}\|u(\tau)\|_{s}^{2} \mathrm{~d} \tau .
$$


First we give bounds on $H^{s}$ norms when the initial condition is also in $H^{s}$.

Lemma 5.2. Choose $0 \leqslant s \leqslant 1$, and suppose that $u_{0} \in H^{s}$ and $f \in L^{2}(0, T ; H)$. Then

$$
\|u(t)\|_{s}^{2}+K_{1+s}(t) \leqslant C\left(\left\|u_{0}\right\|_{s},|f|_{0}\right) .
$$

Furthermore if $u_{0} \in H$ then for any $0<s \leqslant 1 / 2$,

$$
\int_{0}^{t}\|u(s)\|_{1+s} \leqslant c\left(1+|f|_{0}^{2}+\left\|u_{0}\right\|^{2}\right)^{3 / 2}
$$

and if $u_{0} \in H^{s}, 0<s \leqslant 1$, and $f \in L^{2}\left(0, T ; H^{\epsilon}\right)$ for some $0<\epsilon<s$, then

$$
\int_{0}^{t}\|u(\tau)\|_{2+\epsilon} \mathrm{d} \tau \leqslant C\left(\left\|u_{0}\right\|_{s},|f|_{\epsilon}\right)
$$

Proof. Assume that $u_{0} \in H$. By taking the inner product in the Navier-Stokes equations (3.1) with $u$ we obtain, using the orthogonality property (5.1), and the Cauchy-Schwarz and the Poincaré inequalities,

$$
\frac{1}{2} \frac{\mathrm{d}}{\mathrm{d} t}\|u\|^{2}+v\|u\|_{1}^{2}=(f, u) \leqslant c\|f\|^{2}+\frac{v}{2}\|u\|_{1}^{2} .
$$

From this it follows that

$$
\|u(t)\|^{2}+v \int_{0}^{T}\|u(s)\|_{1}^{2} \mathrm{~d} s \leqslant\left\|u_{0}\right\|^{2}+2 c \int_{0}^{t}\|f(s)\|^{2} \mathrm{~d} s,
$$

which is precisely (5.9) for $s=0$.

Now consider the case where $u_{0} \in H^{s}$ for some $s \in(0,1)$. We take the inner product of (3.1) with $A^{s} u$. Since the domain is periodic, $\nabla p$ is perpendicular to any divergence-free square integrable vector, and while we do not know here $a$ priori that $u \in H^{2 s}$, the resulting cancellation of $\nabla p$ can be justified via a Galerkin process. Therefore

$$
\frac{1}{2} \frac{\mathrm{d}}{\mathrm{d} t}\|u\|_{s}^{2}+v\|u\|_{1+s}^{2} \leqslant\left|\left((u \cdot \nabla) u, A^{s} u\right)\right|+\left(f, A^{s} u\right),
$$

and since

$$
\left|\left(f, A^{s} u\right)\right| \leqslant\|f\|\|u\|_{2 s} \leqslant\|f\|\|u\|_{s+1} \leqslant c\|f\|^{2}+\frac{v}{4}\|u\|_{s+1}^{2},
$$

using (5.4) we obtain

$$
\begin{aligned}
\frac{1}{2} \frac{\mathrm{d}}{\mathrm{d} t}\|u\|_{s}^{2}+v\|u\|_{1+s}^{2} & \leqslant c\|u\|_{s}\|u\|_{1}\|u\|_{1+s}+c\|f\|^{2}+\frac{v}{4}\|u\|_{s+1}^{2} \\
& \leqslant c\|u\|_{s}^{2}\|u\|_{1}^{2}+c\|f\|^{2}+\frac{v}{2}\|u\|_{1+s}^{2} .
\end{aligned}
$$

From this we deduce that

$$
\frac{\mathrm{d}}{\mathrm{d} t}\|u\|_{s}^{2}+v\|u\|_{s+1}^{2} \leqslant c\|u\|_{s}^{2}\|u\|_{1}^{2}+c\|f\|^{2} .
$$

Dropping the $v\|u\|_{s+1}^{2}$ term and integrating the inequality gives the bound

$$
\begin{aligned}
\|u(t)\|_{s}^{2} & \leqslant \exp \left(c K_{1}(t)\right)\left(\left\|u_{0}\right\|_{s}^{2}+c \int_{0}^{t}\|f(s)\|^{2} \mathrm{~d} s\right) \\
& \leqslant C\left(\left\|u_{0}\right\|_{s},|f|_{0}\right)
\end{aligned}
$$

from which we deduce that

$$
K_{1+s}(t)=\int_{0}^{t}\|u(\tau)\|_{1+s}^{2} \mathrm{~d} \tau \leqslant C\left(\left\|u_{0}\right\|_{s},|f|_{0}\right) .
$$


For $s=1$ the analysis is simpler, since we have the orthogonality relation (5.2). In this case taking the inner product with $A u$ yields

$$
\frac{1}{2} \frac{\mathrm{d}}{\mathrm{d} t}\|u\|_{1}^{2}+v\|u\|_{2}^{2}=(f, A u) \leqslant c\|f\|^{2}+\frac{v}{2}\|u\|_{2}^{2}
$$

from which bounds on $\|u\|_{1}$ and $K_{2}$ easily follow, and we have shown (5.9).

The additional integral bounds follow using a method due to Foias, Guillopé and Temam [26]. For the first bound (5.10), return to (5.13) (note that the derivation of this inequality does not rely on having $u_{0} \in H^{s}$ for $s>0$ ), which, after relabelling $c$, implies that

$$
\frac{\mathrm{d}}{\mathrm{d} t}\|u\|_{s}^{2}+v\|u\|_{1+s}^{2} \leqslant c\|u\|_{s}^{2}\|u\|_{1}^{2}+c\|f\|^{2} \leqslant c\left(1+\|u\|_{s}^{2}\right)^{2}\left(\|u\|_{1}^{2}+\|f\|^{2}\right) .
$$

Dividing both sides by $\left(1+\|u\|_{s}^{2}\right)^{2}$ and integrating, we obtain

$$
\frac{1}{\left(1+\|u(0)\|_{s}^{2}\right)}-\frac{1}{\left(1+\|u(t)\|_{s}^{2}\right)}+v \int_{0}^{t} \frac{\|u\|_{1+s}^{2}}{\left(1+\|u\|_{s}^{2}\right)^{2}} \mathrm{~d} \tau \leqslant \int_{0}^{t}\left(\|u\|_{1}^{2}+\|f\|^{2}\right) \mathrm{d} \tau
$$

and therefore

$$
\begin{aligned}
\int_{0}^{t} \frac{\|u\|_{1+s}^{2}}{\left(1+\|u\|_{s}^{2}\right)^{2}} \mathrm{~d} \tau & \leqslant c \frac{1}{1+\|u(t)\|_{s}^{2}}+|f|_{0}^{2}+\int_{0}^{t}\|u\|_{1}^{2} \mathrm{~d} \tau \\
& \leqslant c\left(1+|f|_{0}^{2}+\left\|u_{0}\right\|^{2}\right) .
\end{aligned}
$$

Now we use the Cauchy-Schwarz inequality to write

$$
\begin{aligned}
\int_{0}^{t}\|u(\tau)\|_{1+s} \mathrm{~d} \tau & \leqslant\left(\int_{0}^{t} \frac{\|u\|_{1+s}^{2}}{\left(1+\|u\|_{s}^{2}\right)^{2}} \mathrm{~d} \tau\right)^{1 / 2}\left(\int_{0}^{t}\left(1+\|u\|_{s}^{2}\right)^{2} \mathrm{~d} \tau\right)^{1 / 2} \\
& \leqslant c\left(1+|f|_{0}^{2}+\left\|u_{0}\right\|^{2}\right)^{1 / 2}\left(1+\int_{0}^{t}\|u\|_{s}^{4} \mathrm{~d} \tau\right)^{1 / 2} .
\end{aligned}
$$

Assuming that $s \leqslant 1 / 2$ we have, by interpolation, $\|u\|_{s}^{4} \leqslant c\|u\|^{2}\|u\|_{1}^{2}$. Thus

$$
\begin{aligned}
\int_{0}^{t}\|u(\tau)\|_{1+s} \mathrm{~d} \tau & \leqslant c\left(1+|f|_{0}^{2}+\left\|u_{0}\right\|^{2}\right)^{1 / 2}\left(1+c\left(\left\|u_{0}\right\|^{2}+2 c|f|_{0}^{2}\right) K_{1}(t)\right)^{1 / 2} \\
& \leqslant c\left(1+|f|_{0}^{2}+\left\|u_{0}\right\|^{2}\right)^{3 / 2}
\end{aligned}
$$

For the second bound (5.11), taking the inner product of the Navier-Stokes equations with $A^{1+\epsilon} u$ we obtain, using (5.6),

$$
\begin{aligned}
\frac{1}{2} \frac{\mathrm{d}}{\mathrm{d} t}\|u\|_{1+\epsilon}^{2}+v\|u\|_{2+\epsilon}^{2} & \leqslant\left|\left(u \cdot \nabla u, A^{1+\epsilon} u\right)\right|+\left|\left(f, A^{1+\epsilon} u\right)\right| \\
& \leqslant c\|u\|_{1+\epsilon}\|u\|_{1}\|u\|_{2+\epsilon}+c\|u\|_{\epsilon}\|u\|_{2}\|u\|_{2+\epsilon}+\left|\left(A^{\epsilon / 2} f, A^{1+\epsilon / 2} u\right)\right| \\
& \leqslant c\|u\|_{1+\epsilon}\|u\|_{1}\|u\|_{2+\epsilon}+c\|u\|_{\epsilon}\|u\|_{1+\epsilon}^{\epsilon}\|u\|_{2+\epsilon}^{2-\epsilon}+\|f\|_{\epsilon}\|u\|_{2+\epsilon} \\
& \leqslant c\left(\|u\|_{1}^{2}+\|u\|_{\epsilon}^{2 \epsilon}\right)\|u\|_{1+\epsilon}^{2}+c\|f\|_{\epsilon}^{2}+\frac{v}{2}\|u\|_{2+\epsilon}^{2} .
\end{aligned}
$$

We first obtain a bound on $\int_{0}^{t}\|u(\tau)\|_{2+\epsilon} \mathrm{d} \tau$. Taking the inner product of the Navier-Stokes equations with $A^{1+\epsilon} u$ we obtain, using (5.6),

$$
\begin{aligned}
\frac{1}{2} \frac{\mathrm{d}}{\mathrm{d} t}\|u\|_{1+\epsilon}^{2}+v\|u\|_{2+\epsilon}^{2} & \leqslant\left|\left(u \cdot \nabla u, A^{1+\epsilon} u\right)\right|+\left|\left(f, A^{1+\epsilon} u\right)\right| \\
& \leqslant 2\left\|\left|D u\|D u\| D A^{\epsilon} u\right|\right\|_{L^{1}}+2\left\|\left|u\left\|D^{2} u\right\| D A^{\epsilon} u\right|\right\|_{L^{1}}+\left|\left(A^{\epsilon / 2} f, A^{1+\epsilon / 2} u\right)\right| \\
& \leqslant c\|u\|_{1+\epsilon}\|u\|_{1}\|u\|_{2+\epsilon}+c\|u\|_{\epsilon}\|u\|_{1+\epsilon}^{\epsilon}\|u\|_{2+\epsilon}^{2-\epsilon}+\|f\|_{\epsilon}\|u\|_{2+\epsilon} \\
& \leqslant c\left(\|u\|_{1}^{2}+\|u\|_{\epsilon}^{2 / \epsilon}\right)\|u\|_{1+\epsilon}^{2}+c\|f\|_{\epsilon}^{2}+\frac{v}{2}\|u\|_{2+\epsilon}^{2}
\end{aligned}
$$


and where we have also used the interpolation inequality $\|u\|_{2} \leqslant c\|u\|_{1+\epsilon}^{\epsilon}\|u\|_{2+\epsilon}^{1-\epsilon}$ to obtain the second term in the third line. The final inequality implies that

$$
\frac{\mathrm{d}}{\mathrm{d} t}\|u\|_{1+\epsilon}^{2}+v\|u\|_{2+\epsilon}^{2} \leqslant c\left(\|u\|_{1}^{2}+\|u\|_{\epsilon}^{2 / \epsilon}+\|f\|_{\epsilon}^{2}\right)\left(1+\|u\|_{1+\epsilon}^{2}\right)^{1+\beta}
$$

for any $\beta>0$. Therefore after dividing both sides by $\left(1+\|u\|_{1+\epsilon}^{2}\right)^{1+\beta}$ and integrating, we conclude from (5.12) that, by interpolation,

$$
\begin{aligned}
\int_{0}^{t} \frac{\|u\|_{2+\epsilon}^{2}}{\left(1+\|u\|_{1+\epsilon}^{2}\right)^{1+\beta}} \mathrm{d} s \leqslant & \frac{1}{\beta\left(1+\|u(t)\|_{1+\epsilon}^{2}\right)^{\beta}} \\
& +c \int_{0}^{t}\left(\|u\|_{1}^{2}+\|u\|^{2(1-\epsilon) / \epsilon}\|u\|_{1}^{2}+\|f\|_{\epsilon}^{2}\right) \mathrm{d} s \\
\leqslant & C\left(\left\|u_{0}\right\|\right)\left(1+|f|_{\epsilon}^{2}+K_{1}(t)\right) .
\end{aligned}
$$

Now, by the Hölder inequality, we can write

$$
\int_{0}^{t}\|u\|_{2+\epsilon} \mathrm{d} s \leqslant\left(\int_{0}^{t} \frac{\|u\|_{2+\epsilon}^{2}}{\left(1+\|u\|_{1+\epsilon}^{2}\right)^{1+\beta}} \mathrm{d} s\right)^{1 / 2}\left(\int_{0}^{t}\left(1+\|u\|_{1+\epsilon}^{2}\right)^{1+\beta} \mathrm{d} s\right)^{1 / 2}
$$

Let $\beta=\frac{s-\epsilon}{1+\epsilon}$. By the interpolation inequality, since $\epsilon<s$, we have

$$
\begin{aligned}
\int_{0}^{t}\left(1+\|u\|_{1+\epsilon}^{2}\right)^{1+\beta} \mathrm{d} \tau & \leqslant \int_{0}^{t}\left(1+\|u\|^{2\left(1-\frac{1+\epsilon}{1+s}\right)}\|u\|_{1+s}^{2 \frac{1+\epsilon}{1+s}}\right)^{1+\beta} \mathrm{d} \tau \\
& \leqslant c+c \sup _{0<\tau<t}\|u(\tau)\|^{2(s-\epsilon) /(1+s)} \int_{0}^{t}\|u\|_{1+s}^{2} \mathrm{~d} \tau \\
& \leqslant C\left(\left\|u_{0}\right\|_{s},|f|_{0}\right),
\end{aligned}
$$

by (5.15). Therefore we conclude that

$$
\int_{0}^{t}\|u(\tau)\|_{2+\epsilon} \mathrm{d} \tau \leqslant C\left(\left\|u_{0}\right\|_{s},|f|_{0}\right)\left(1+|f|_{\epsilon}^{2}+\left\|u_{0}\right\|^{2}\right)^{\frac{1}{2}}
$$

We now give bounds on the decay of the $H^{s}$ norms when $u_{0} \in H$. Note that the following gives an alternative proof of the decay rate of weak solutions obtained by Giga and Mirakawa [29], which was obtained using the semigroup approach of Kato and Fujita [45], and which is used in the proof of uniqueness of particle trajectories for 2D weak solutions due to Dashti and Robinson [18].

Lemma 5.3. Assume that $u_{0} \in H$ and that $f \in L^{2}\left(0, T ; H^{l}\right)$, with $l=0$ when $0 \leqslant s \leqslant 1$ and $l=s-1$ otherwise. Then there exists a constant $c$ independent of $u_{0}$ such that, for any $t>t_{0}$

$\|u(t)\|_{s}^{2}+\int_{t_{0}}^{t}\|u(\tau)\|_{1+s}^{2} \mathrm{~d} \tau \leqslant\left\{\begin{array}{lr}c t_{0}^{-s}\left(|f|_{0}^{2}+\left\|u_{0}\right\|^{2}\right) \quad \text { for } & 0<s \leqslant 1, \\ c t_{0}^{-s}\left(|f|_{l}+|f|_{0}^{2}+\left\|u_{0}\right\|^{2}\right)^{2} & \text { for } 1<s<2 .\end{array}\right.$

Proof. To obtain (5.17) in the case $s=1$, we take the inner product of the Navier-Stokes equations with $A u$. Since we are in a periodic domain $((u \cdot \nabla) u, A u)=0$, and we obtain

$$
\frac{1}{2} \frac{\mathrm{d}}{\mathrm{d} t}\|u\|_{1}^{2}+v\|u\|_{2}^{2} \leqslant|(f, A u)| \leqslant c\|f\|^{2}+\frac{v}{2}\|u\|_{2}^{2}
$$

and therefore

$$
\frac{\mathrm{d}}{\mathrm{d} t}\|u\|_{1}^{2}+v\|u\|_{2}^{2} \leqslant c\|f\|^{2}
$$


After dropping $v\|u\|_{2}^{2}$ and integrating we obtain, for $0<\sigma<t \leqslant T$,

$$
\|u(t)\|_{1}^{2} \leqslant\|u(\sigma)\|_{1}^{2}+c|f|_{0}^{2}
$$

recalling the notation (4.7). Integrating with respect to $\sigma$ between 0 and $t \leqslant T$ we obtain, from (5.12),

$$
\begin{aligned}
t\|u(t)\|_{1}^{2} & \leqslant \int_{0}^{T}\|u(\sigma)\|_{1}^{2} \mathrm{~d} \sigma+t c|f|_{0}^{2} \\
& \leqslant c\left(|f|_{0}^{2}+\left\|u_{0}\right\|^{2}\right) .
\end{aligned}
$$

Therefore the bound (5.17) follows for $s=1$, and that for $0<s<1$ by interpolation between this bound and (5.12). We note also that

$$
v \int_{t_{0}}^{T}\|u(t)\|_{2}^{2} \mathrm{~d} t \leqslant c t_{0}^{-1}\left(|f|_{0}^{2}+\left\|u_{0}\right\|^{2}\right)
$$

again one can interpolate to deduce that for $0 \leqslant l \leqslant 1$ we have

$$
v \int_{t_{0}}^{T}\|u(t)\|_{1+l}^{2} \mathrm{~d} t \leqslant c t_{0}^{-l}\left(|f|_{0}^{2}+\left\|u_{0}\right\|^{2}\right)
$$

Now consider $1<s<2$, and write $s=1+l$. Taking the inner-product of the NavierStokes equations with $A^{s} u$ we obtain, using (5.6), and Young's inequality,

$$
\begin{aligned}
\frac{1}{2} \frac{\mathrm{d}}{\mathrm{d} t}\|u\|_{s}^{2}+v\|u\|_{1+s}^{2} & \leqslant c\|u\|_{s}\|u\|_{1}\|u\|_{1+s}+c\|u\|_{l}\|u\|_{2}\|u\|_{1+s}+\left(f, A^{s} u\right) \\
& \leqslant c\|u\|_{s}^{2}\|u\|_{1}^{2}+c\|u\|_{l}^{2}\|u\|_{2}^{2}++\frac{v}{2}\|u\|_{1+s}^{2}+c\|f\|_{l}^{2}+\frac{3 v}{8}\|u\|_{1+s}^{2} .
\end{aligned}
$$

Thus

$$
\frac{\mathrm{d}}{\mathrm{d} t}\|u\|_{s}^{2}+v\|u\|_{1+s}^{2} \leqslant c\|u\|_{s}^{2}\|u\|_{1}^{2}+c\|u\|_{l}^{2}\|u\|_{2}^{2}+c\|f\|_{l}^{2} .
$$

Choose $t_{0}<t$ and integrate over $\left[t_{0} / 2+\sigma, t\right]$ to give

$$
\begin{aligned}
\|u(t)\|_{s}^{2} & \leqslant\left\|u\left(t_{0} / 2+\sigma\right)\right\|_{s}^{2}+c\left(\sup _{t_{0} / 2+\sigma \leqslant \tau \leqslant t}\|u(\tau)\|_{1}^{2}\right) \int_{t_{0} / 2+\sigma}^{t}\|u(\tau)\|_{s}^{2} \mathrm{~d} \tau \\
& +c\left(\sup _{t_{0} / 2+\sigma \leqslant \tau \leqslant t}\|u(\tau)\|_{l}^{2}\right) \int_{t_{0} / 2+\sigma}^{t}\|u(\tau)\|_{2}^{2} \mathrm{~d} \tau+c|f|_{l}^{2} .
\end{aligned}
$$

It follows from (5.17) that

$$
\|u(t)\|_{s}^{2} \leqslant\left\|u\left(t_{0} / 2+\sigma\right)\right\|_{s}^{2}+c t_{0}^{-(1+l)}\left(|f|_{0}^{2}+\left\|u_{0}\right\|^{2}\right)^{2}+c|f|_{l}^{2}
$$

recalling the notation (4.7). We now integrate with respect to $\sigma$ in the interval $\left[0, t-t_{0} / 2\right]$ to find, since $s<2$,

$\left(t-t_{0} / 2\right)\|u(t)\|_{s}^{2} \leqslant c \int_{t_{0} / 2}^{t}\|u(\sigma)\|_{s}^{2} \mathrm{~d} \sigma+\left(t-t_{0} / 2\right) c t_{0}^{-s}\left(|f|_{0}^{2}+\left\|u_{0}\right\|^{2}\right)^{2}+c|f|_{l}^{2}$.

Since $t>t_{0}$ we obtain, using (5.17) for $0<s<1$,

$$
\|u(t)\|_{s}^{2} \leqslant c t_{0}^{-s}\left(|f|_{l}+|f|_{0}^{2}+\left\|u_{0}\right\|^{2}\right)^{2} .
$$

The related bound on $\int_{t_{0}}^{t}\|u\|_{1+s}^{2} \mathrm{~d} \tau$ then follows from integrating (5.21). 


\subsection{Bounds on differences of solutions: Lipschitz continuity}

Now we consider the difference of two solutions of the Navier-Stokes equations, showing that the solution mapping is Lipschitz continuous between various Sobolev spaces. Let $(u(x, t), p(x, t))$ and $(v(x, t), q(x, t))$ be solutions of (3.1) and (3.2) with initial condition $u_{0}$ and $v_{0}$, and driving forces $\zeta$ and $\xi$, respectively; furthermore assume that both $u_{0}$ and $v_{0}$ are in $H$. The difference function $w(t)=u(t)-v(t)$ satisfies

$\frac{\partial w}{\partial t}+v A w+(u \cdot \nabla) w+(w \cdot \nabla) u-(w \cdot \nabla) w+\nabla(p-q)=r, \quad w(0)=u_{0}-v_{0}$,

where $r=\zeta-\xi$. By analogy with the notation $K_{s}(t)$ for the integral of the $H^{s}$ norm of $u$, we define

$$
W_{s}(t):=\int_{0}^{t}\|w(\tau)\|_{s}^{2} \mathrm{~d} \tau
$$

First we show that $u_{0} \mapsto u(t)$ is Lipschitz from $H^{s}$ into $H^{s}$, and that the solution mapping is Lipschitz from $H^{s}$ into $L^{2}\left(0, T ; H^{s+1}\right)$.

Lemma 5.4. Let $u_{0}, v_{0} \in H^{s}$ and $\zeta, \xi \in L^{2}(0, T ; H)$. Then for $0 \leqslant s \leqslant 1$,

$\|w(t)\|_{s}^{2}+W_{1+s}(t) \leqslant C\left(\left\|u_{0}\right\|,|\zeta|_{0},|r|_{0},\|w(0)\|\right)\left(\left\|u_{0}-v_{0}\right\|_{s}^{2}+|r|_{0}^{2}\right)$.

Proof. Let $s \in[0,1)$. To bound $\|w(t)\|_{s}$, we take the inner product of (5.22) with $A^{s} w$. Since the inner product of the gradient of a scalar function with a divergence free vector field is zero in a periodic domain, we obtain, noting that $2 s<1+s$,

$$
\begin{aligned}
\frac{1}{2} \frac{\mathrm{d}}{\mathrm{d} t}\|w\|_{s}^{2}+v\|w\|_{1+s}^{2} \leqslant & \left|\left((w \cdot \nabla) u, A^{s} w\right)\right|+\left|\left((u \cdot \nabla) w, A^{s} w\right)\right|+\left|\left((w \cdot \nabla) w, A^{s} w\right)\right| \\
& +c\|r\|^{2}+\frac{v}{4}\|w\|_{1+s}^{2} .
\end{aligned}
$$

For $s=0$ two of the terms on the right-hand side vanish (using the orthogonality property (5.1)). Then using (5.3),

$$
\begin{aligned}
\frac{1}{2} \frac{\mathrm{d}}{\mathrm{d} t}\|w\|^{2}+v\|w\|_{1}^{2} & \leqslant \mid((w \cdot \nabla) u, w)) \mid+c\|r\|^{2}+\frac{v}{4}\|w\|_{1}^{2} \\
& \leqslant c\|w\|\|u\|_{1}\|w\|_{1}+c\|r\|^{2}+\frac{v}{4}\|w\|_{1}^{2} \\
& \leqslant c\|w\|^{2}\|u\|_{1}^{2}+c\|r\|^{2}+\frac{v}{2}\|w\|_{1}^{2}
\end{aligned}
$$

and therefore

$$
\frac{\mathrm{d}}{\mathrm{d} t}\|w\|^{2}+v\|w\|_{1}^{2} \leqslant c\|w\|^{2}\|u\|_{1}^{2}+c\|r\|^{2}
$$

Dropping $v\|w\|_{1}^{2}$ and integrating we obtain the bound

$$
\|w(t)\|^{2} \leqslant \exp \left(K_{1}(T)\right)\left(\|w(0)\|^{2}+c|r|_{0}^{2}\right) \text { for a.e. } t \in[0, T]
$$

Having this, going back to (5.25) and integrating again, we obtain from the bound on $K_{1}$ in (5.9),

$$
\begin{aligned}
v W_{1}(t) & =v \int_{0}^{T}\|w(t)\|_{1}^{2} \mathrm{~d} t \\
& \leqslant\|w(0)\|^{2}+c \int_{0}^{T}\|r(s)\|^{2} \mathrm{~d} s+c \sup _{0 \leqslant t \leqslant T}\|w(t)\|^{2} K_{1}(T) \\
& \leqslant C\left(\left\|u_{0}\right\|,|\zeta|_{0}\right)\left(\|w(0)\|^{2}+|r|_{0}^{2}\right) .
\end{aligned}
$$


For $0<s<1$ we have, using (5.4),

$$
\begin{aligned}
\left|\left((w \cdot \nabla) u, A^{s} w\right)\right| & \leqslant\|w\|_{s}\|u\|_{1}\|w\|_{s+1} \\
& \leqslant c\|w\|_{s}^{2}\|u\|_{1}^{2}+\frac{v}{8}\|w\|_{s+1}^{2} ;
\end{aligned}
$$

for the second term we use (5.4) once again along with the Sobolev interpolation inequalities $\|u\|_{s} \leqslant\|u\|^{1-s}\|u\|_{1}^{s}$ and $\|w\|_{1} \leqslant\|w\|_{s}^{s}\|w\|_{1+s}^{1-s}$,

$$
\begin{aligned}
\left|\left((u \cdot \nabla) w, A^{s} w\right)\right| & \leqslant\|u\|_{s}\|w\|_{1}\|w\|_{s+1} \\
& \leqslant\|u\|^{1-s}\|u\|_{1}^{s}\|w\|_{s}^{s}\|w\|_{1+s}^{2-s} \quad \text { (Sobolev interpolation) } \\
& \leqslant c\|u\|^{2(1-s) / s}\|u\|_{1}^{2}\|w\|_{s}^{2}+\frac{v}{8}\|w\|_{s+1}^{2} ;
\end{aligned}
$$

and for the third term we use (5.5),

$$
\begin{aligned}
\left|\left((w \cdot \nabla) w, A^{s} w\right)\right| & \leqslant c\|w\|^{1 / 2}\|w\|_{s}^{1 / 2}\|w\|_{1}^{1 / 2}\|w\|_{s+1}^{3 / 2} \\
& \leqslant c\|w\|^{2}\|w\|_{s}^{2}\|w\|_{1}^{2}+\frac{v}{8}\|w\|_{s+1}^{2} .
\end{aligned}
$$

Together these imply that

$$
\frac{\mathrm{d}}{\mathrm{d} t}\|w\|_{s}^{2}+v\|w\|_{1+s}^{2} \leqslant c\left(\|u\|_{1}^{2}+\|u\|^{2(1-s) / s}\|u\|_{1}^{2}+\|w\|^{2}\|w\|_{1}^{2}\right)\|w\|_{s}^{2}+c\|r\|^{2} .
$$

Therefore, dropping $v\|w\|_{1+s}^{2}$ and integrating over $[0, t]$, we have

$$
\|w(t)\|_{s}^{2} \leqslant \exp \left(c \int_{0}^{t}\left(\|u\|_{1}^{2}+\|u\|^{2(1-s) / s}\|u\|_{1}^{2}+\|w\|^{2}\|w\|_{1}^{2}\right) \mathrm{d} \tau\right)\left(\|w(0)\|_{s}^{2}+c|r|_{0}^{2}\right) \text {. }
$$

Using the bounds in (5.9), (5.26) and (5.27) to obtain pointwise and integral bounds on $u$ and $w$ we conclude that

$$
\|w(t)\|_{s}^{2} \leqslant C\left(\left\|u_{0}\right\|,|\zeta|_{0},|r|_{0},\|w(0)\|\right)\left(\left\|u_{0}-v_{0}\right\|_{s}^{2}+|r|_{0}^{2}\right) .
$$

This also implies, by integrating (5.28), that

$$
W_{1+s}(T)=\int_{0}^{T}\|w(\tau)\|_{1+s}^{2} \mathrm{~d} \tau \leqslant C\left(\left\|u_{0}\right\|,|\zeta|_{0},|r|_{0},\|w(0)\|\right)\left(\left\|u_{0}-v_{0}\right\|_{s}^{2}+|r|_{0}^{2}\right) .
$$

For $s=1$ we use (5.5) repeatedly and eventually obtain

$$
\frac{\mathrm{d}}{\mathrm{d} t}\|D w\|^{2}+v\|A w\|^{2} \leqslant c\left(\|u\|_{1}^{2}+\|u\|^{2}\|u\|_{1}^{2}\right)\|D w\|^{2}+c\|u\|_{2}^{2}\|w\|^{2} .
$$

Since both $\|u\|_{2}^{2}\|w\|^{2}$ and the expression in the parentheses in the right-hand side are integrable when $u_{0} \in H^{1}$, the estimate (5.23) follows for $s=1$ after integrating in the same way as above.

A more refined analysis shows that in fact for $t>0$ the map $u_{0} \mapsto u(t)$ is Lipschitz continuous from $H$ into more regular $H^{s}$ spaces, as is the solution mapping from $H$ into $L^{2}\left(t, T ; H^{s+1}\right)$.

Lemma 5.5. Let $u_{0}, v_{0} \in H, \zeta, \xi \in L^{2}(0, T ; H)$ and $r \in L^{2}\left(0, T ; H^{l}\right)$ with $l=0$ for $0 \leqslant s \leqslant 1$ and $l=s-1$ for $1<s<2$. Then for $0 \leqslant s<2$ and any $t>t_{0}$,

$\|w(t)\|_{s}^{2}+\int_{t_{0}}^{T}\|w(\tau)\|_{1+s}^{2} \mathrm{~d} \tau \leqslant t_{0}^{-s} C\left(\left\|u_{0}\right\|,|\zeta|_{0},|r|_{0},\|w(0)\|\right)\left(\left\|u_{0}-v_{0}\right\|^{2}+|r|_{l}^{2}\right)$. 
Proof. We now return to the case $u_{0}, v_{0} \in H$, and consider the case $s=1$. We drop $v\|A w\|^{2}$ in (5.32) integrate with respect to time between $\sigma+t_{0} / 2$ and $t$, with $0<\sigma<t$ and $t \geqslant t_{0}$, to obtain, using (5.17),

$$
\begin{aligned}
\|w(t)\|_{1}^{2} \leqslant & c\left\|w\left(\sigma+t_{0} / 2\right)\right\|_{1}^{2} \\
& +c \sup _{\tau \in\left[\sigma+t_{0} / 2, t\right]}\|u(\tau)\|_{1}^{2}\left(1+\|u(\tau)\|^{2}\right) \int_{0}^{t}\|w\|_{1}^{2} \mathrm{~d} \tau \\
& +c \sup _{\tau \in[0, T]}\|w(\tau)\|^{2} \int_{\sigma+t_{0} / 2}^{t}\|u\|_{2}^{2} \mathrm{~d} \tau \\
\leqslant & c\left\|w\left(\sigma+t_{0} / 2\right)\right\|_{1}^{2}+t_{0}^{-1} C\left(\left\|u_{0}\right\|,\|w(0)\|,\|f\|_{L^{2}(0, T ; H)}\right)\|w(0)\|^{2}
\end{aligned}
$$

Integrating the above inequality with respect to $\sigma$ over $\left(0, t-t_{0} / 2\right)$ yields

$$
t\|w(t)\|_{1}^{2} \leqslant C\left(\left\|u_{0}\right\|,\|w(0)\|,\|f\|_{L^{2}(0, T ; H)}\right)\|w(0)\|^{2},
$$

since $t_{0} \leqslant t$ was arbitrary. From the above inequality and (5.26) we conclude (5.33) for $0<s \leqslant 1$ by interpolation. This also implies, by integrating (5.32) and interpolation, that for any $0 \leqslant s \leqslant 1$,

$t_{0}^{s} \int_{t_{0}}^{T}\|w(\tau)\|_{1+s}^{2} \mathrm{~d} \tau \leqslant C\left(\left\|u_{0}\right\|,|\zeta|_{0},|r|_{0},\|w(0)\|\right)\left(\left\|u_{0}-v_{0}\right\|^{2}+|r|_{0}^{2}\right)$.

Now we have the estimates needed to obtain the bounds on $\|w(t)\|_{s}$ with $s \in(1,2)$. Taking the inner product of (5.22) with $A^{s} w$ and using (5.6), we can write

$$
\begin{aligned}
\frac{\mathrm{d}}{\mathrm{d} t}\|w\|_{s}^{2}+v\|w\|_{1+s}^{2} & \leqslant c\left(\|u\|_{s}^{2}+\|w\|_{s}^{2}\right)\|w\|_{1}^{2}+c\left(\|u\|_{s-1}^{2}+\|w\|_{s-1}^{2}\right)\|w\|_{2}^{2} \\
& +c\|w\|_{s}^{2}\|u\|_{1}^{2}+c\|w\|_{s-1}^{2}\|u\|_{2}^{2}+c\|r\|_{s-1}^{2} .
\end{aligned}
$$

Now dropping $v\|w\|_{1+s}^{2}$ and integrating between $\sigma+t_{0} / 2$ and $t$ with $0<\sigma<t$ and $t>t_{0}$, we obtain, using (5.17), (5.34) and (5.23)

$$
\begin{aligned}
\|w(t)\|_{s}^{2} \leqslant & \left\|w\left(\sigma+t_{0} / 2\right)\right\|_{s}^{2}+c|r|_{s-1}^{2} \\
& +c \sup _{\tau \in\left[\sigma+t_{0} / 2, t\right]}\|w(\tau)\|_{1}^{2} \int_{\sigma+t_{0} / 2}^{t}\left(\|u\|_{s}^{2}+\|w\|_{s}^{2}\right) \mathrm{d} \tau \\
& +c \sup _{\tau \in\left[\sigma+t_{0} / 2, t\right]}\left(\|u\|_{s-1}^{2}+\|w\|_{s-1}^{2}\right) \int_{\sigma+t_{0} / 2}^{t}\|w\|_{2}^{2} \mathrm{~d} \tau \\
& +c \sup _{\tau \in\left[\sigma+t_{0} / 2, t\right]}\|u(\tau)\|_{1}^{2} \int_{\sigma+t_{0} / 2}^{t}\|w\|_{s}^{2} \mathrm{~d} \tau \\
& +c \sup _{\tau \in\left[\sigma+t_{0} / 2, t\right]}\|w(\tau)\|_{s-1}^{2} \int_{\sigma+t_{0} / 2}^{t}\|u\|_{2}^{2} \mathrm{~d} \tau \\
\leqslant & \left\|w\left(\sigma+t_{0} / 2\right)\right\|_{s}^{2}+t_{0}^{-s} C\left(\left\|u_{0}\right\|,|\zeta|_{0},|r|_{0},\|w(0)\|\right)\left(\|w(0)\|^{2}+|r|_{s-1}^{2}\right)
\end{aligned}
$$

After integrating with respect to $\sigma$ between 0 and $t-t_{0} / 2$, we end up with

$$
t_{0}^{s}\|w(t)\|_{s}^{2} \leqslant C\left(\left\|u_{0}\right\|,|\zeta|_{0},|r|_{0},\|w(0)\|\right)\left(\|w(0)\|^{2}+|r|_{s-1}^{2}\right) .
$$

for $t>t_{0}>0$. 


\subsection{Differentiability}

We show here that the solution mapping $u_{0} \mapsto u(t)$ is differentiable as a map from $H$ into $H^{s}$ for any $0 \leqslant s<2$.

Lemma 5.6. Let $u\left(x, t ; u_{0}\right)$ be a solution of the Navier-Stokes equations with $u(0)=u_{0} \in H$. Then for each $t>0$ the mapping $u_{0} \mapsto u(t)$ is differentiable from $H$ into $H^{s}$ for all $0 \leqslant s<2$, with $U=\frac{\partial u}{\partial u_{0}}\left(u_{0}-v_{0}\right)$ the solution of the linearized equation

$$
\frac{\mathrm{d} U}{\mathrm{~d} t}+v A U+B(u, U)+B(U, u)=0, \quad \text { with } \quad U(0)=u_{0}-v_{0} .
$$

Proof. Assume that $v$ is the solution of the Navier-Stokes equations with $v(0)=v_{0}$. The function $\theta=u-v-U$ satisfies

$$
\frac{\mathrm{d} \theta}{\mathrm{d} t}+v A \theta+B(u, \theta)+B(\theta, u)-B(w, w)=0, \quad \theta(0)=0
$$

where $w=u-v$. To show the required differentiability we need to show

$$
\sup _{\left\|u_{0}-v_{0}\right\|<\varepsilon} \frac{\|u(x, t)-v(x, t)-U(x, t)\|_{s}}{\left\|u_{0}-v_{0}\right\|} \rightarrow 0 \quad \text { as } \varepsilon \rightarrow 0 .
$$

Let $s \in[0,1)$. Taking the inner product of (5.36) with $A^{s} \theta$ and after finding appropriate estimates for the nonlinear terms as before, we obtain

$$
\begin{aligned}
\frac{1}{2} \frac{\mathrm{d}}{\mathrm{d} t}\|\theta\|_{s}^{2}+v\|\theta\|_{1+s}^{2} \leqslant & c^{\prime}\|u\|_{s}\|\theta\|_{1}\|\theta\|_{1+s}+c\|\theta\|_{s}\|u\|_{1}\|\theta\|_{1+s} \\
& +c\|w\|_{s}\|w\|_{1}\|\theta\|_{1+s} .
\end{aligned}
$$

Here both $c$ and $c^{\prime}$ are constants independent of $u, w, \theta$ and $c^{\prime}=0$ if $s=0$, but is nonzero otherwise. By the interpolation inequality

$$
\|u\|_{s}\|\theta\|_{1}\|\theta\|_{1+s} \leqslant\|u\|^{1-s}\|u\|_{1}^{s}\|\theta\|_{s}^{s}\|\theta\|_{1+s}^{2-s}
$$

we have, from the Hölder inequality,

$\frac{\mathrm{d}}{\mathrm{d} t}\|\theta\|_{s}^{2}+v\|\theta\|_{1+s}^{2} \leqslant c^{\prime}\|u\|^{2(1-s) / s}\|u\|_{1}^{2}\|\theta\|_{s}^{2}+c\|u\|_{1}^{2}\|\theta\|_{s}^{2}+c\|w\|_{s}^{2}\|w\|_{1}^{2}$.

In the case $s=0$ we deduce, by first bounding $\|\theta(t)\|^{2}$ using Gronwall, and then integrating to bound $\int_{0}^{t}\|\theta(\tau)\|_{1}^{2} \mathrm{~d} \tau$, that

$$
\|\theta(t)\|^{2}+\int_{0}^{t}\|\theta(\tau)\|_{1}^{2} \mathrm{~d} \tau \leqslant C\left(\left\|u_{0}\right\|,\left\|w_{0}\right\|\right)\left\|w_{0}\right\|^{4} .
$$

Now we consider $0<s<1$. Dropping $v\|\theta\|_{1+s}^{2}$, integrating using Gronwall's inequality, we find that

$$
\begin{aligned}
\|\theta(t)\|_{s}^{2} & \leqslant\left(\int_{t_{0} / 2+\sigma}^{t} c^{\prime \prime}\|u(r)\|_{1}^{2} \mathrm{~d} r\right)\left(\left\|\theta\left(t_{0} / 2+\sigma\right)\right\|_{s}^{2}+c \int_{t_{0} / 2+\sigma}^{t}\|w\|_{s}^{2}\|w\|_{1}^{2} \mathrm{~d} r\right) \\
& \leqslant C\left(\left\|u_{0}\right\|,\left\|w_{0}\right\|,|f|_{0}\right)\left\|\theta\left(t_{0} / 2+\sigma\right)\right\|_{s}^{2}+C\left(\left\|u_{0}\right\|,\left\|w_{0}\right\|,|f|_{0}\right) t_{0}^{-s}\left\|w_{0}\right\|^{4},
\end{aligned}
$$

from which it follows that

$$
\|\theta(t)\|_{s}^{2} \leqslant t_{0}^{-s} C\left(\left\|u_{0}\right\|,\left\|w_{0}\right\|,|f|_{0}\right)\left\|w_{0}\right\|^{4}, \quad s \in(0,1) .
$$

for $t>t_{0}$. Having the bound on $\|\theta\|_{s}$, going back to (5.37) and integrating, yields

$$
\int_{t_{0}}^{T}\|\theta\|_{s+1}^{2} \mathrm{~d} \tau \leqslant t_{0}^{-s} C\left(\left\|u_{0}\right\|,\left\|w_{0}\right\|,|f|_{0}\right)\left\|w_{0}\right\|^{4}, \quad s \in(0,1) .
$$


For $s=1$ we have, using (5.5) repeatedly, and Young's inequality $\frac{\mathrm{d}}{\mathrm{d} t}\|\theta\|_{1}^{2}+v\|\theta\|_{2}^{2} \leqslant c\|u\|^{2}\|u\|_{1}^{2}\|\theta\|_{1}^{2}+c\|\theta\|\|\theta\|_{1}\|u\|_{1}\|u\|_{2}+c\|w\|\|w\|_{1}^{2}\|w\|_{2}$.

Integrating the above inequality, and bounding $\|u\|,\|\theta\|$, and $\|w\|$ using (5.9), (5.38), and (5.23), give

$$
\begin{aligned}
\|\theta(t)\|_{1}^{2} \leqslant & \left\|\theta\left(t_{0} / 2+\sigma\right)\right\|_{1}^{2}+c\|u\|_{1}^{2} \int_{t_{0} / 2+\sigma}^{t}\|\theta\|_{1}^{2} \\
& +c\|u\|_{1}\left(\int\|\theta\|_{1}^{2}\right)^{1 / 2}\left(\int\|u\|_{2}^{2}\right)^{1 / 2}\left\|w_{0}\right\|^{2} \\
& +c\|w\|_{1}\left(\int\|w\|_{1}^{2}\right)^{1 / 2}\left(\int\|w\|_{2}^{2}\right)^{1 / 2}\left\|w_{0}\right\| \\
\leqslant & \left\|\theta\left(t_{0} / 2+\sigma\right)\right\|_{1}^{2}+t_{0}^{-1} C\left(\left\|u_{0}\right\|,\left\|w_{0}\right\|,|f|_{0}\right)\left\|w_{0}\right\|^{4},
\end{aligned}
$$

where we have also used (5.33). From this we obtain

$$
\|\theta(t)\|_{1}^{2} \leqslant t_{0}^{-1} C\left(\left\|u_{0}\right\|,\left\|w_{0}\right\|,|f|_{0}\right)\left\|w_{0}\right\|^{4} \text {. }
$$

Now consider any $s \in(1,2)$. Taking the inner product of (5.36) with $A^{s} \theta$ and using (5.6), we can write

$$
\begin{aligned}
\frac{\mathrm{d}}{\mathrm{d} t}\|\theta\|_{s}^{2}+v\|\theta\|_{1+s}^{2} & \leqslant c\|\theta\|_{s}^{2}\|u\|_{1}^{2}+c\|\theta\|_{s-1}^{2}\|u\|_{2}^{2} \\
& +c\|u\|_{s}^{2}\|\theta\|_{1}^{2}+c\|u\|_{s-1}^{2}\|\theta\|_{2}^{2} \\
& +c\|w\|_{s}^{2}\|w\|_{1}^{2}+c\|w\|_{s-1}^{2}\|w\|_{2}^{2} .
\end{aligned}
$$

Again, dropping $v\|\theta\|_{1+s}^{2}$, integrating twice similarly as above, and using (5.17), (5.33), (5.39) and (5.40) we obtain

$$
\|\theta(t)\|_{s}^{2} \leqslant t_{0}^{-s} C\left(\left\|u_{0}\right\|,\left\|w_{0}\right\|,|f|_{0}\right)\left\|w_{0}\right\|^{4}, \quad 1<s<2,
$$

for $t>t_{0}>0$, and the required differentiability follows.

\subsection{Differentiability of the Lagrangian mapping: proof of lemma 3.7}

We have

$$
\frac{\mathrm{d} z}{\mathrm{~d} t}=u\left(z, t ; u_{0}\right)
$$

Letting $U=\frac{\partial u}{\partial u_{0}}\left(u_{0}-v_{0}\right)$ and $Z=\frac{\partial z}{\partial u_{0}}\left(u_{0}-v_{0}\right)$, the linearized form of the above equation with respect to the initial velocity field is as follows

$$
\frac{\mathrm{d} Z}{\mathrm{~d} t}=U(z, t)+\nabla u(z, t) Z, \quad \text { with } \quad Z(0)=0
$$

where $U$ satisfies the linearized Navier-Stokes equations (5.35). To prove the required differentiability, we show that

We can write

$$
\sup _{\left\|u_{0}-v_{0}\right\|_{s}<\varepsilon} \frac{|z(t)-y(t)-Z(t)|}{\left\|u_{0}-v_{0}\right\|_{s}} \rightarrow 0 \quad \text { as } \varepsilon \rightarrow 0 .
$$

$$
\begin{aligned}
\frac{\mathrm{d}|z-y-Z|}{\mathrm{d} t} \leqslant & |u(z, t)-v(y, t)-U(z, t)-\nabla u(z, t) Z| \\
\leqslant & |u(y, t)-v(y, t)-U(y, t)| \\
& +|u(z, t)-u(y, t)-\nabla u(z, t)(z-y)| \\
& +|U(y, t)-U(z, t)|+|\nabla u(z, t)(z-y-Z)|
\end{aligned}
$$


with $z(0)-y(0)-Z(0)=0$. By the Sobolev embedding theorem we have

$$
|u(y, t)-v(y, t)-U(y, t)| \leqslant c\|u(t)-v(t)-U(t)\|_{1+s}
$$

and

$$
|\nabla u(z, t)(z-y-Z)| \leqslant c\|u\|_{2+\epsilon}|z-y-Z|
$$

with some $\epsilon<s$. By the mean value theorem (which makes sense here since the solution $u(x, t)$ is smooth in space for any $t>0)$, with $\varsigma$ and $\eta$ two points on the line connecting $z(t)$ and $y(t)$, we can write

$$
\begin{aligned}
& \mid u(z, t)-u(y, t)-\nabla u(z, t)(z-y) \mid \\
&=\left|\left(\begin{array}{c}
\nabla u_{1}(\varsigma, t)-\nabla u_{1}(z, t) \\
\nabla u_{2}(\eta, t)-\nabla u_{2}(z, t)
\end{array}\right)(z-y)\right| \\
& \leqslant c\|u\|_{2+\epsilon}|z-y|^{1+\epsilon / 2}
\end{aligned}
$$

where in the last line we have used the following inequality ([60], lemma 7.26)

$$
|D u(z)-D u(y)| \leqslant c\|u\|_{2+\epsilon}|z-y|^{\epsilon_{1}} .
$$

for any $\epsilon_{1}<\epsilon$. Finally, since $\theta=u-v-U$ and therefore $\|U\|_{1+s} \leqslant\|\theta\|_{1+s}+\|w\|_{1+s}$, we have

$$
|U(z, t)-U(y, t)| \leqslant c\|U\|_{1+s}|z-y|^{\epsilon} \leqslant c\left(\|\theta\|_{1+s}+\|w\|_{1+s}\right)|z-y|^{\epsilon} .
$$

Hence

$$
\begin{aligned}
\frac{\mathrm{d}}{\mathrm{d} t}|z-y-Z| & \leqslant c\|u\|_{2+\epsilon}|y-z-Z| \\
& +\|\theta\|_{1+s}+c\|u\|_{2+\epsilon}|z-y|^{1+\epsilon / 2}+c\left(\|\theta\|_{1+s}+\|w\|_{1+s}\right)|z-y|^{\epsilon}
\end{aligned}
$$

implying that

$$
\begin{aligned}
|z(t)-y(t)-Z(t)| & \leqslant \exp \left(\int_{0}^{t}\|u\|_{2+\epsilon} \mathrm{d} \tau\right)\left(\int_{0}^{t}\|\theta\|_{1+s} \mathrm{~d} \tau\right. \\
& +c \sup _{\tau \in[0, t]}|z(\tau)-y(\tau)|^{1+\epsilon / 2} \int_{0}^{t}\|u\|_{2+\epsilon} \mathrm{d} \tau \\
& \left.+c \sup _{\tau \in[0, t]}|z(\tau)-y(\tau)|^{\epsilon} \int_{0}^{t}\left(\|\theta\|_{1+s}+\|w\|_{1+s}\right) \mathrm{d} \tau\right) .
\end{aligned}
$$

Now, to bound $\int_{0}^{t}\|\theta\|_{1+s} \mathrm{~d} \tau$ appropriately, we note that having $u_{0}, v_{0} \in H^{s}$, from (5.37)—after integration with respect to time and using (5.23)—we can obtain

$$
\begin{aligned}
\|\theta(t)\|_{s}^{2} & \leqslant c \exp \left(C\left(\left\|u_{0}\right\|,|f|_{0}\right) \int_{0}^{t}\|u\|_{1}^{2} \mathrm{~d} \tau\right) \int_{0}^{t}\|w\|_{s}^{2}\|w\|_{1}^{2} \\
& \leqslant C\left(\left\|u_{0}\right\|,\|w(0)\|,|f|_{0}\right)\|w(0)\|^{2}\|w(0)\|_{s}^{2}
\end{aligned}
$$

and therefore

$$
\int_{0}^{t}\|\theta(\tau)\|_{1+s}^{2} \mathrm{~d} \tau \leqslant C\left(\left\|u_{0}\right\|,\|w(0)\|,|f|_{0}\right)\|w(0)\|^{2}\|w(0)\|_{s}^{2} .
$$

This gives the required differentiability, because by (5.23), (5.11) and (3.11) we have

$$
\begin{aligned}
& |z(t)-y(t)-Z(t)| \\
& \quad \leqslant C\left(\left\|u_{0}\right\|_{s},\|w(0)\|_{s},|f|_{\epsilon}\right)\left(\left\|u_{0}-v_{0}\right\|+\left\|u_{0}-v_{0}\right\|_{s}^{\epsilon / 2}+\left\|u_{0}-v_{0}\right\|_{s}^{\epsilon}\right)\left\|u_{0}-v_{0}\right\|_{s} .
\end{aligned}
$$




\section{Conclusions}

The main contributions of this paper are twofold:

(A) We develop an abstract framework in which to study inverse problems for functions, given finite data (section 2).

(B) We employ the abstract framework to study the integration of data with the PDE models arising in fluid mechanics (section 3).

Regarding item (A) there are three main results. In theorem 2.1 and corollary 2.2 we describe an infinite-dimensional version of the Bayes theorem which will be useful in applications; this theorem gives conditions under which the posterior measure is absolutely continuous with respect to the prior measure and identifies the Radon-Nikodym derivative between them. In theorem 2.5 we prove a well-posedness result for the posterior measure, demonstrating continuity of the measure with respect to changes in the data; such results are, of course, sensitive to the choice of probability metric and we are able to work in the Hellinger metric, implying continuity of expectations of all polynomially bounded functions in the data, a very strong conclusion. In theorem 2.7 we study MAP estimators on function space and show that the same conditions which give rise to a well-defined posterior measure also give rise to a well-defined maximization problem for the probability, using straightforward ideas from the calculus of variations.

Regarding item (B) we study four problems in detail, all based around determining the solution of the Navier-Stokes equations in two dimensions. We study problems with or without model error and where the data involve direct pointwise observations of the velocity field (Eulerian) or indirect observations of it via the positions of particle tracers (Lagrangian). We show that the posterior measure is well defined and absolutely continuous with respect to the prior measure, identifying the Radon-Nikodym derivative as the exponential of a quadratic form measuring model-data mismatch. Roughly speaking, the posterior measure gives high probability to functions which lead to a small model-data mismatch, and low probability otherwise, all stated relative to the prior probability measure; this prior will specify quantitative information, such as the mean, as well as qualitative information such as the regularity expected from the function of interest. Results of this type are stated in theorems 3.4, 3.8, 3.11 and 3.14 for the four problems studied. The lemmas in these sections also show that the abstract theorems 2.5 and 2.7 both apply to all four model problems and that, hence, there are continuity results for the posterior measure with respect to data and variational methods are well defined, both in the function space setting.

Looking forward we anticipate three primary directions in which this work can be taken. First the existence of a form of well posedness for the Bayesian inverse problem on function space suggests the possibility of deriving approximation theories for the finitedimensional approximations of the posterior required in computational practice. Second, demonstrating that the abstract framework can be applied to study different inverse problems in differential equations, such as determination of constitutive models in porous media or medical tomography applications. And third to use the ideal solution encapsulated in the posterior measure as a benchmark against which to test, study and improve existing methods used in practical data assimilation.

\section{Acknowledgments}

The authors are grateful to L Borcea and C M Elliott for helpful suggestions concerning this material. They are also grateful to two anonymous referees and an anonymous journal board member for advice leading to a significant restructuring of the material herein. AMS 
is grateful to EPSRC, ERC and ONR for financial support; MD and SLC are grateful to the Warwick Postgraduate Fellowship fund and to EPSRC (respectively) for support of their graduate studies.

\section{References}

[1] Apte A, Hairer M, Stuart A M and Voss J 2007 Sampling the posterior: an approach to non-Gaussian data assimilation Physica D 230 50-64

[2] Apte A, Jones C K R T and Stuart A M 2008 A Bayesian approach to Lagrangian data assimilation Tellus A $60336-47$

[3] Apte A, Jones C K R T, Stuart A M and Voss J 2008 Data assimilation: mathematical and statistical perspectives Int. J. Numer. Methods Fluids 56 1033-46

[4] Bengtsson T, Snyder C and Nychka D 2003 Toward a nonlinear ensemble filter for high-dimensional systems J. Geophys. Res. 1088775

[5] Bennett A F 2002 Inverse Modeling of the Ocean and Atmosphere (Cambridge: Cambridge University Press)

[6] Bennett A F and Budgell W D 1987 Ocean data assimilation and the MOAN filter: spatial regularity J. Phys. Oceanograph. 17 1583-601

[7] Berliner L M 2001 Monte Carlo based ensemble forecasting Stat. Comput. 11 269-75

[8] Beskos A and Stuart A M 2007 MCMC methods for sampling function space Proc. ICIAM

[9] Beskos A, Roberts G, Stuart A M and Voss J An MCMC method for diffusion bridges Stochast. Dyn. 8 319-50

[10] Bogachev V I 1998 Gaussian Meausures (Providence, RI: American Mathematical Society)

[11] Calvetti D and Somersalo E 2008 Large-scale statistical parameter estimation in complex systems with an application to metabolic models Multiscale Model. Simul. 5 1333-66

[12] Chemin J-Y and Lerner N 1995 Flot de champs de vecteurs non-Lipschitziens et équations de Navier-Stokes $J$. Differ. Equ. $121314-28$

[13] Cotter S L, Dashti M, Robinson J C and Stuart A M 2009 MCMC Methods on Function Space and Applications to Fluid Mechanics in preparation

[14] Cohn S E 1997 An introduction to estimation theory J. Met. Soc. Japan 75 257-88

[15] Courtier P, Anderson E, Heckley W, Pailleux J, Vasiljevic D, Hamrud M, Hollingworth A, Rabier F and Fisher M 1998 The ECMWF implementation of three-dimensional variational assimilation (3d-var) Q. J. R. Met. Soc. 124 1783-808

[16] Dacorogna B 1989 Direct Methods in the Calculus of Variations (Berlin: Springer)

[17] DaPrato G and Zabczyk J 1992 Stochastic Equations in Infinite Dimensions (Cambridge: Cambridge University Press)

[18] Dashti M and Robinson J C 2009 A simple proof of uniqueness of the particle trajectories for solutions of the Navier-Stokes equations Nonlinearity $22735-46$

[19] Derber J C 1989 A variational continuous assimilation technique Mon. Weather Rev $1172437-46$

[20] Dostert P, Efendiev Y, Hou T Y and Luo W 2006 Coarse-grain Langevin algorithms for dynamic data integration J. Comput. Phys. 217 123-42

[21] Engl H K, Hanke M and Neubauer A 1996 Regularization of Inverse Problems (Dordrecht: Kluwer)

[22] Dudley R M 2002 Real Analysis and Probability (Cambridge: Cambridge University Press)

[23] Evensen G 2007 Data Assimilation: the Ensemble Kalman Filter (New York: Springer)

[24] Farmer C L 2007 Bayesian field theory applied to scattered data interpolation and inverse problems Algorithms for Approximation ed A Iske and J Leveseley (Berlin: Springer) pp 147-66

[25] Farmer C L 2005 Geological modelling and reservoir simulation Mathematical Methods and Modelling in Hydrocarbon Exploration and Production ed A Iske and T Randen (Berlin: Springer-Verlag)

[26] Foias C, Guillopé C and Temam R 1981 New a priori estimates for Navier-Stokes equations in dimension 3 Comm. Part. Differ. Equ. 6 329-59

[27] Franklin J N 1970 Well-posed stochastic extensions of ill-posed linear problems J. Math. Anal. Appl. 31 682-716

[28] Gibbs A L and Su F E 2002 On choosing and bounding probability metrics Int. Stat. Rev. 70 419-35

[29] Giga Y and Miyakawa T 1985 Solutions in Lr of the Navier-Stokes initial value problem Arch. Ration. Mech. Anal. $89267-81$

[30] Hagelberg C R, Bennett A F and Jones D A 1996 Local existence results for the generalized inverse of the vorticity equation in the plane Inverse Problems 12 437-54

[31] Hairer M 2009 private communication 
[32] Hairer M, Stuart A M, Voss J and Wiberg P 2005 Analysis of SPDEs arising in path sampling: part I. The Gaussian case Commun. Math. Sci. 3 587-603

[33] Hairer M, Stuart A M and Voss J 2007 Analysis of SPDEs arising in path sampling: part I. The non-Gaussian case Ann. Appl. Prob. 3 587-603

[34] Hein T 2009 On Tikhonov regularization in Banach spaces-optimal convergence rate results Appl. Anal. 88 653-67

[35] Hey J G and Trefethen A E 2003 The data deluge: an e-science perspective Grid Computing-Making the Global Infrastructure a Reality ed F Berman, G C Fox and A J G Hey (New York: Wiley) chapter 36, pp 809-24

[36] Hofinger A and Pikkarainen H K 2007 Convergence rates for the Bayesian approach to linear inverse problems Inverse Problems 23 2469-84

[37] Ide K, Courtier P, Ghil M and Lorenc A C 1997 Unified notation for data assimilation: operational, sequential and variational J. Met. Soc. Japan 75 181-9

[38] Ide K, Kuznetsov L and Jones C K R T 2002 Lagrangian data assimilation for point-vortex system $J$. Turbulence 353

[39] Kaipio J and Somersalo E 2004 Statistical and Computational Inverse Problems (Berlin: Springer)

[40] Kaipio J P and Somersalo E 2000 Statistical inversion and Monte Carlo sampling methods in electrical impedance tomography Inverse Problems 16 1487-522

[41] Kaipio J P and Somersalo E 2007 Statistical inverse problems: discretization, model reduction and inverse crimes J. Comput. Appl. Math. 198 493-504

[42] Kaltenbacher B, Schöpfer F and Schuster T 2009 Iterative methods for nonlinear ill-posed problems in Banach spaces: convergence and applications to parameter identification problems Inverse Problems 25065003

[43] Kallenberg O 2002 Foundations of Modern Probability 2nd edn (New York: Springer)

[44] Kalnay E 2003 Atmospheric Modeling, Data Assimilation and Predictability (Cambridge: Cambridge University Press)

[45] Kato T and Fujita H 1962 On the nonstationary Navier-stokes system Rend. Sem. Mat. Univ. Padova 32 243-60

[46] Kinderlehrer D and Stampacchia G 1980 An Introduction to Variational Inequalities and Their Applications (New York: Academic)

[47] Kuznetsov L, Ide K and Jones C K R T 2003 A method for assimilation of Lagrangian data Mon. Weather Rev. 1312247

[48] Lassas M, Saksman E and Siltanen S 2009 Discretization-invariant Bayesian inversion and Besov space priors Inverse Problems 3 87-122

[49] Lassas M and Siltanen S 2004 Can one use total variation prior for edge-preserving Bayesian inversion Inverse Problems 20 1537-64

[50] Lorenc A C 1986 Analysis methods for numerical weather prediction Q. J. R. Met. Soc. 112 1177-94

[51] Lorenc A C and Payne T 2007 4D-Var and the butterfly effect: Statistical four-dimensional data assimilation for a wide range of scales Q. J. Royal Met. Soc. 133 607-14

[52] Neubauer A 2009 On enhanced convergence rates for tikhonov regularization of nonlinear ill-posed problems in banach spaces Inverse Problems 25065009

[53] Nichols N K 2003 Data assimilation: aims and basic concepts Data Assimilation for the Earth System ed R Swinbank, V Shutyaev and W A Lahoz (Netherlands: Kluwer) pp 9-20

[54] Nodet M 2006 Variational assimilation of Lagrangian data in oceanography Inverse Problems 22 245-63

[55] Nodet M 2005 Mathematical modelling and assimilation of Lagrangian data in oceanography PhD Thesis University of Nice

[56] Ott E, Hunt B R, Szunyogh I, Zimin A V, Kostelich E J, Corazza M, Kalnay E, Patil D J and Yorke J A 2004 A local ensemble Kalman filter for atmospheric data assimilation Tellus A 56 237-77

[57] Palmer T N, Doblas-Reyes F J, Weisheimer A, Shutts G J, Berner J and Murphy J M 2009 Towards the probabilistic earth-system model in preparation

[58] Papoulis A 1984 Probability, Random Variables and Stochastic Processes (New York: McGraw-Hill)

[59] Ramsay J O and Silverman B W 2005 Functional Data Analysis (Berlin: Springer)

[60] Renardy M and Rogers R C 2004 An Introduction to Partial Differential Equations 2nd edn (New York: Springer)

[61] Roberts G O and Rosenthal J 2001 Optimal scaling for various Metropolis-hastings algorithms Stat. Sci. $16351-67$

[62] Robinson J C 2001 Infinite Dimensional Dynamical Systems (Cambridge: Cambridge University Press)

[63] Rudin L I, Osher S and Fatemi E 1992 Nonlinear total variation based noise removal algorithms Physica D 60 259-68 
[64] Talagrand O 2009 Evaluation of assimilation algorithms Data Assimilation: Making Sense of Observations ed W Lahoz et al (Berlin: Springer)

[65] Talagrand O and Courtier P 1987 Variational assimilation of meteorological observations with the adjoint vorticity equation. I: Theory Q. J. R. Met. Soc. 113 1311-28

[66] Tarantola A 2005 Inverse Problem Theory and Methods for Model Parameter Estimation (PA, USA: SIAM)

[67] Temam R 1983 Navier-Stokes equations and nonlinear functional analysis Regional Conference Series in Applied Mathematics SIAM (Philadelphia)

[68] White L W 1993 A study of uniqueness for the initialization problem for Burgers' equation J. Math. Anal. Appl. 172 412-31 